Key words: Discontinuous Petrov Galerkin, incompressible flow, Navier-Stokes equations, adaptive finite elements

AMS subject classification: $65 \mathrm{~N} 30,35 \mathrm{~L} 15$

\title{
A Discontinuous Petrov-Galerkin Methodology for Adaptive Solutions to the Incompressible Navier-Stokes Equations
}

\author{
Nathan V. Roberts ${ }^{\mathrm{a}, *}$, Leszek Demkowicz ${ }^{\mathrm{b}}$, Robert Moser ${ }^{\mathrm{b}}$ \\ ${ }^{a}$ Argonne Leadership Computing Facility, Argonne National Laboratory, Argonne, IL, USA. \\ ${ }^{b}$ Institute for Computational Engineering and Sciences, The University of Texas at Austin, Austin, TX, USA.
}

\begin{abstract}
The discontinuous Petrov-Galerkin methodology with optimal test functions (DPG) of Demkowicz and Gopalakrishnan $[18,20]$ guarantees the optimality of the solution in an energy norm, and provides several features facilitating adaptive schemes. Whereas Bubnov-Galerkin methods use identical trial and test spaces, Petrov-Galerkin methods allow these function spaces to differ. In DPG, test functions are computed on the fly and are chosen to realize the supremum in the inf-sup condition; the method is equivalent to a minimum residual method. For well-posed problems with sufficiently regular solutions, DPG can be shown to converge at optimal rates-the inf-sup constants governing the convergence are mesh-independent, and of the same order as those governing the continuous problem [48]. DPG also provides an accurate mechanism for measuring the error, and this can be used to drive adaptive mesh refinements.

We employ DPG to solve the steady incompressible Navier-Stokes equations in two dimensions, building on previous work on the Stokes equations, and focusing particularly on the usefulness of the approach for automatic adaptivity starting from a coarse mesh. We apply our approach to a manufactured solution due to Kovasznay as well as the lid-driven cavity flow, backward-facing step, and flow past a cylinder problems.
\end{abstract}

\section{Introduction}

\subsection{Motivation}

Typical solutions of incompressible flow problems involve both fine- and large-scale phenomena, so that a uniform finite element mesh of sufficient granularity will at best be wasteful of computational resources, and at worst be infeasible because of resource limitations. Thus adaptive mesh refinements are desirable. In industry, the adaptivity schemes used are often ad hoc, requiring a domain expert to predict features of the solution. A badly chosen mesh may cause the code to take considerably longer to converge, or fail to converge altogether. Typically, the Navier-Stokes solve will be just one component in an optimization loop, which means that any failure requiring human intervention is costly.

* Corresponding author.

Email addresses: nvroberts@anl.gov, leszek@ices.utexas.edu,rmoser@ices.utexas.edu. 
Our aim, therefore, is to develop a solver for the incompressible Navier-Stokes equations that provides robust adaptivity starting from a coarse mesh. ${ }^{1}$ By robust, we mean both that the solver always converges to a solution in predictable time, and that the adaptive scheme is independent of the problem-no special expertise is required for adaptivity. In each of the adaptive experiments in the present work, we begin with a mesh that simply captures the geometry, perform some preliminary refinements to ensure that elements are roughly isotropic, and proceed with the automatic adaptivity algorithm described below.

\subsection{Approach}

The cornerstone of our approach will be the discontinuous Petrov-Galerkin with optimal test functions (DPG) finite element methodology recently developed by Leszek Demkowicz and Jay Gopalakrishnan [18, 20]. Whereas BubnovGalerkin methods use the same function space for both test and trial functions, Petrov-Galerkin methods allow the spaces for test and trial functions to differ. In DPG, the test functions are computed on the fly and are chosen to minimize the residual. For a very broad class of well-posed problems, DPG offers provably optimal convergence rates with a modest stability constant - the "inf-sup" constants governing the convergence are mesh-independent, and of the same order as those governing the continuous problem [48]. In some of our experiments, DPG not only achieves the optimal rates, but gets very close to the best solution available in the discrete space. DPG also provides an accurate mechanism for measuring the error, and this can be used to drive adaptive mesh refinements.

Previously, we have studied DPG applied to the Stokes problem in some detail, with theoretical results predicting optimal rates of convergence, and numerical results that appear to show even more: it appears that we asymptotically approach the best approximation error available in the discrete space [48]. We began with Stokes because the Stokes equations are more susceptible than Navier-Stokes to rigorous mathematical analysis; our strategy is to use the theory developed for Stokes to guide the practical application to Navier-Stokes. Because of the success with the Stokes equations and their close relationship to the incompressible Navier-Stokes equations, we are optimistic that DPG can achieve good results with the latter as well.

Central to our study of these problems has been the use and further development of Camellia [47], a toolbox we developed for solving DPG problems which uses Sandia's Trilinos library of packages [29]. At present, Camellia supports arbitrary 1D meshes, 2D meshes of triangles and quads, and 3D meshes of hexahedra, provides mechanisms for easy specification of DPG variational forms, supports $h$ - and $p$ - refinements, and employs a distributed stiffness matrix and solution representation, among other features. In the future, we plan to enhance support for meshes of arbitrary spatial dimension, and add support for space-time elements and a distributed mesh representation.

\subsection{Literature Review: Adaptive Navier-Stokes}

The application of adaptive mesh refinement to incompressible flow problems is far from new. Here, we mention a few relevant references for finite element methods, spectral element methods, and least squares methods. DPG is a

\footnotetext{
${ }^{1}$ Ultimately, we would like to produce such a solver for incompressible Navier-Stokes in arbitrary dimensions, for a range of Reynolds numbers limited only by the numerical precision of the machine. The scope of the present work is more modest: we limit ourselves to steady two-dimensional flows; the largest Reynolds number that we employ is $10^{4}$. We restrict ourselves thus both to maintain a focused discussion and because transient and three-dimensional flows impose additional implementation challenges. In upcoming work, we plan to address transient and three-dimensional problems.
} 
Petrov-Galerkin finite-element method (FEM), a generalization of the classical (Bubnov) Galerkin method. As early as 1993, Oden presented an approach for $h p$-adaptive FEM for the incompressible Navier-Stokes equations [42].

Spectral element methods (SEM) employ basis functions with global support, in contrast to FEM, which employ basis functions with support limited to the element. Karniadakis and Sherwin have produced a compendium of $h p$-adaptive SEM for computational fluid dynamics [31]. Galerkin FEM and SEM for incompressible flow problems require careful selection of velocity and pressure spaces; unstable discretizations can result in locking or nonconvergence. This contrasts with the present work, in which we use equal-order discretizations for velocity and pressure, and for smooth solutions we obtain optimal rates of convergence in both variables. Moreover, DPG allows the discretizations for velocity and pressure to be chosen independently.

Least-squares finite and spectral element methods (LSFEM and LSSEM) employ formulations that minimize a residual, typically in the $L^{2}$ norm. Like DPG, least-squares methods allow independent selection of velocity and pressure discretizations. Recently Ozcelikkale and Sert have applied $h p$-adaptive LSSEM to model problems in 2D incompressible flow [43]. Like least-squares, DPG is a minimum-residual method-in fact, DPG can be understood as a least-squares method which minimizes the DPG energy norm. In contrast to classical least-squares methods, DPG's energy norm can be prescribed by appropriate selection of the norm on the test space, allowing it to avoid some of the problems classically exhibited by least-squares, such as over-diffusivity.

\subsection{The Discontinuous Petrov-Galerkin Method with Optimal Test Functions: Previous Work}

We begin with a short historical review of the DPG method. By a discontinuous Galerkin (DG) method, we mean one that allows test and/or trial functions that are not globally conforming; by a Petrov-Galerkin method, we mean one that allows the test and trial spaces to differ. In 2002, Bottasso et al. introduced a method [4, 5], also called DPG. Like our DPG method, theirs used an "ultra-weak" variational formulation (moving all derivatives to test functions) and replaced the numerical fluxes used in DG methods to "glue" the elements together with new independent unknowns defined on element interfaces. The idea of optimal testing was introduced by Demkowicz and Gopalakrishnan in 2009 [18], which is distinguished by an on-the-fly computation of an approximation to a set of test functions that are optimal in the sense that they realize the supremum in the inf-sup condition. These can then be shown to guarantee minimization of the residual in the dual norm. In 2009-2010, a flurry of numerical experimentation followed, including applications to convection-dominated diffusion [20], wave propagation [56], elasticity [6], thin-body (beam and shell) problems [39], and the Stokes problem [49]. The wave propagation paper also introduced the concept of an optimal test norm, whose selection makes the energy norm identical to the norm of interest on the trial space. In 2010, Demkowicz and Gopalakrishnan proved the convergence of the method for the Laplace equation [19], and Demkowicz and Heuer developed a systematic approach to the selection of a test space norm for singularly perturbed problems [21]. In 2011, Bui-Thanh et al. [9] developed a unified analysis of DPG problems by means of Friedrichs' systems. Our analysis for the Stokes problem, presented in [48], builds on the existence of trace spaces and proceeds along a more classical path, connecting to Banach's theory of closed operators.

Some work has been done on nonlinear problems as well. Very early on, Chan, Demkowicz, and Roberts solved the 1D Burgers and compressible Navier-Stokes equations by applying DPG to the linearized problem [13]. Moro et al. have applied their related HDPG method to the 2D Burgers equation; a key difference in their work is that they apply 
DPG to the nonlinear problem, using optimization techniques to minimize the DPG residual. Chan, Demkowicz, and Moser continued the compressible Navier-Stokes effort [12], using an analysis of convection-diffusion problems to develop test norms for high Reynolds number flows. Bui-Thanh and Ghattas developed a PDE-constrained optimization approach to DPG [10]; whereas our present approach applies DPG to the linearized problem, Bui-Thanh and Ghattas's approach unifies the treatment of linear and nonlinear problems, allowing direct application of DPG to nonlinear problems, as well as providing an iterative solution technique for DPG problems generally. Our present work employs only direct solvers; as with any matrix system, it is possible to use standard iterative solvers to solve the system that arises from DPG. Recently, Wieners and Wohlmuth have constructed general preconditioners for saddle-point systems of a sort that arise from DPG formulations [54]; these offer another path for iterative solution of DPG problems.

Most DPG analysis assumes that the optimal test functions are computed exactly, but in practice we must approximate them. Gopalakrishnan and Qiu have shown that for the Laplace equation and linear elasticity, for sufficiently high-order approximations ${ }^{2}$ of the test space, optimal $h$-convergence rates are maintained [26].

\subsection{Paper Outline}

The structure of this paper is as follows. In Section 2, we briefly derive the abstract method, then describe the steps required to apply the method, deriving formulations for Stokes and Navier-Stokes and setting up the discrete problem to be solved. In Section 3, we perform several numerical experiments to demonstrate the convergence and the adaptive power of the method: we study a manufactured solution due to Kovasznay, the lid-driven cavity problem, the backward-facing step problem, and the flow past a cylinder problem. We conclude in Section 4.

\section{DPG Step by Step}

\subsection{Abstract DPG Method}

We will now briefly derive DPG, motivating it as a minimum residual method. Suppose that $U$ is the trial space, and $V$ the test space (both Hilbert) for a well-posed variational problem $b(u, v)=l(v)$. Writing this in the operator form $B u=l$, where $B: U \rightarrow V^{\prime}$, we seek to minimize the residual for the discrete space $U_{h} \subset U$ :

$$
u_{h}=\underset{u_{h} \in U_{h}}{\arg \min } \frac{1}{2}\left\|B u_{h}-l\right\|_{V^{\prime}}^{2} .
$$

Now, the dual space $V^{\prime}$ is not especially easy to work with; we would prefer to work with $V$ itself. Recalling that the Riesz operator $R_{V}: V \rightarrow V^{\prime}$ defined by

$$
\left\langle R_{V} v, \delta v\right\rangle=(v, \delta v)_{V}, \quad \forall \delta v \in V
$$

where $\langle\cdot, \cdot\rangle$ denotes the duality pairing between $V^{\prime}$ and $V$, is an isometry— that is, $\left\|R_{V} v\right\|_{V^{\prime}}=\|v\|_{V}$ — we can rewrite the term we want to minimize as a norm in $V$ :

$$
\frac{1}{2}\left\|B u_{h}-l\right\|_{V^{\prime}}^{2}=\frac{1}{2}\left\|R_{V}^{-1}\left(B u_{h}-l\right)\right\|_{V}^{2}=\frac{1}{2}\left(R_{V}^{-1}\left(B u_{h}-l\right), R_{V}^{-1}\left(B u_{h}-l\right)\right)_{V} .
$$

\footnotetext{
${ }^{2} k_{\text {test }}=k_{\text {trial }}+N$, where $N$ is the number of space dimensions, by $k_{\text {test }}$ we mean the polynomial order of the basis functions for the test space, and by $k_{\text {trial }}$ we mean the order for the $L^{2}$ bases in the trial space.
} 
The first-order optimality condition requires that the Gâteaux derivative of (2.1) be equal to zero for minimizer $u_{h}$; we have

$$
\left(R_{V}^{-1}\left(B u_{h}-l\right), R_{V}^{-1} B \delta u_{h}\right)_{V}=0, \quad \forall \delta u_{h} \in U_{h}
$$

By the definition of $R_{V}$, the preceding equation is equivalent to

$$
\left\langle B u_{h}-l, R_{V}^{-1} B \delta u_{h}\right\rangle=0 \quad \forall \delta u_{h} \in U_{h} .
$$

Now, if we identify $v_{\delta u_{h}}=R_{V}^{-1} B \delta u_{h}$ as a test function, we can rewrite (2.2) as

$$
b\left(u_{h}, v_{\delta u_{h}}\right)=l\left(v_{\delta u_{h}}\right) .
$$

Note that the last equation is exactly the original variational form, tested with a special function $v_{\delta u_{h}}$ that corresponds to $\delta u_{h} \in U_{h}$; we call $v_{\delta u_{h}}$ an optimal test function. The DPG method is then to solve the problem $b\left(u_{h}, v_{\delta u_{h}}\right)=$ $l\left(v_{\delta u_{h}}\right)$ with optimal test functions $v_{\delta u_{h}} \in V$ that solve the problem

$$
\left(v_{\delta u_{h}}, \delta v\right)_{V}=\left\langle R_{V} v_{\delta u_{h}}, \delta v\right\rangle=\left\langle B \delta u_{h}, \delta v\right\rangle=b\left(\delta u_{h}, \delta v\right), \quad \forall \delta v \in V
$$

In standard conforming methods, test functions are continuous over the entire domain, which would mean that solving (2.3) would require computations on the global mesh, making the method impractical. In DPG, we use test functions that are discontinuous across elements, so that (2.3) becomes a local problem-that is, it can be solved element by element. Of course, solving (2.3) would still require inversion of the infinite-dimensional Riesz map; we approximate this by using an "enriched" test space $V_{h}$ of polynomial order higher than that of the trial space $U_{h}$. Note that the test functions $v_{\delta u_{h}}$ immediately give rise to a hermitian positive definite stiffness matrix; if $\left\{e_{i}\right\}$ is a basis for $U_{h}$, we have:

$$
b\left(e_{i}, v_{e_{j}}\right)=\left(v_{e_{i}}, v_{e_{j}}\right)_{V}={\overline{\left(v_{e_{j}}, v_{e_{i}}\right)}}_{V}=\overline{b\left(e_{j}, v_{e_{i}}\right)} .
$$

It should be pointed out that we have not made any assumptions about the inner product on $V$. An important point is that by an appropriate choice of test space inner product, the induced energy norm on the trial space can be made to coincide with the norm of interest [56]; DPG then delivers the best approximation error in that norm. In practice this optimal test space inner product is approximated by a "localizable" inner product, and DPG delivers the best approximation error up to a mesh-independent constant. That is,

$$
\left\|u-u_{h}\right\|_{U} \leq \frac{M}{\gamma_{D P G}} \inf _{w_{h} \in U_{h}}\left\|u-w_{h}\right\|_{U}
$$

where $M=O(1)$ and $\gamma_{D P G}$ is mesh-independent, and $\gamma_{D P G}$ is of the order of inf-sup constants for the strong operator and its adjoint (see [48]). We therefore say that DPG is automatically stable, modulo any error in solving for the test functions $v_{\delta u_{h}}$.

It is a relatively simple matter, when desired, to enforce local conservation-that is, an element-wise property that corresponds to a (mass) conservation law-by means of Lagrange multipliers. This was first noted by Moro et al. [38]. This is often useful in the context of practical fluid problems. We have not yet, however, explored combining DPG with local conservation in any great detail. In the present work, local conservation was not enforced, but we have measured mass production in each experiment-for converged adaptive solutions, mass production is negligibly small. 


\subsection{Stokes on a Fixed Mesh with Straight Edges}

We begin with the classical strong form of the Stokes equations:

$$
\begin{aligned}
-\nabla p+\mu \Delta \boldsymbol{u} & =\boldsymbol{f} \\
\nabla \cdot \boldsymbol{u} & =0,
\end{aligned}
$$

on some domain $\Omega$, where $\boldsymbol{u}$ is the velocity, $p$ is the pressure, and $\boldsymbol{f}$ is a vector-valued forcing function. By appropriate non-dimensionalization, we may take $\mu=1$ without loss of generality. We assume that Dirichlet boundary conditions $\boldsymbol{u}=\boldsymbol{g}$ are specified on the boundary $\partial \Omega$. We also assume a zero-mean condition on the pressure:

$$
\int_{\Omega} p=0
$$

The steps to solve the system using DPG are as follows:

1. Determine the variational formulation.

2. Specify boundary conditions.

3. Specify the test space inner product.

4. Define discrete trial and test spaces (including the mesh).

5. Compute optimal test functions.

6. Assemble the stiffness matrix.

7. Solve the global problem.

We treat each of these in turn.

Variational Formulation. In DPG, our standard practice is to use an ultra-weak variational formulation, a first order system in which all derivatives have been moved onto test functions. Generally speaking, there are several ways of doing this. Here, we derive the velocity-gradient-pressure (VGP) formulation for the Stokes equations. We begin by introducing $\boldsymbol{\sigma}=\nabla \boldsymbol{u}$ to get a first-order system:

$$
\begin{aligned}
-\nabla p+\nabla \cdot \boldsymbol{\sigma} & =\boldsymbol{f}, \\
\boldsymbol{\sigma}-\nabla \boldsymbol{u} & =0, \\
\nabla \cdot \boldsymbol{u} & =0 .
\end{aligned}
$$

Testing with $(\boldsymbol{v}, q, \boldsymbol{\tau})$, and integrating by parts on a mesh $\Omega_{h}$ with skeleton $\Gamma_{h}$, we have

$$
\begin{aligned}
(\boldsymbol{\sigma}-p \boldsymbol{I}, \nabla \boldsymbol{v})_{\Omega_{h}}-\langle(\boldsymbol{\sigma}-p \boldsymbol{I}) \boldsymbol{n}, \boldsymbol{v}\rangle_{\Gamma_{h}} & =(\boldsymbol{f}, \boldsymbol{v})_{\Omega_{h}} \\
(\boldsymbol{u}, \nabla q)_{\Omega_{h}}-\langle\boldsymbol{u} \cdot \boldsymbol{n}, q\rangle_{\Gamma_{h}} & =0 \\
(\boldsymbol{\sigma}, \boldsymbol{\tau})_{\Omega_{h}}+(\boldsymbol{u}, \nabla \cdot \boldsymbol{\tau})_{\Omega_{h}}-\langle\boldsymbol{u}, \boldsymbol{\tau} \boldsymbol{n}\rangle_{\Gamma_{h}} & =\mathbf{0}
\end{aligned}
$$


In the ultra-weak formulation, since we take no derivatives of our trial variables, we take our so-called field variables to be in $L^{2}\left(\Omega_{h}\right)$, which means that we cannot speak of these on the mesh skeleton $\Gamma_{h}$. We therefore introduce new variables $\widehat{\boldsymbol{t}}_{n} \stackrel{\text { def }}{=}(\boldsymbol{\sigma}-p \boldsymbol{I}) \boldsymbol{n}$ and $\widehat{\boldsymbol{u}}$. The hat notation indicates that these variables are only defined on the mesh skeleton- $\widehat{\boldsymbol{t}}_{n} \in \boldsymbol{H}^{-1 / 2}\left(\Gamma_{h}\right)$ is a trace of an $H$ (div) function (which we call a flux variable), and $\widehat{\boldsymbol{u}} \in \boldsymbol{H}^{1 / 2}\left(\Gamma_{h}\right)$ is a trace of an $\boldsymbol{H}^{1}$ function. Defining group variables $u=(\boldsymbol{u}, p, \boldsymbol{\sigma}), \widehat{u}=\left(\widehat{\boldsymbol{u}}, \widehat{\boldsymbol{t}}_{n}\right)$ and $v=(\boldsymbol{v}, q, \boldsymbol{\tau})$, we arrive at our ultra-weak variational formulation:

$$
\begin{aligned}
b((u, \widehat{u}), v)=(\boldsymbol{\sigma} & -p \boldsymbol{I}, \nabla \boldsymbol{v})_{\Omega_{h}}-\left\langle\widehat{\boldsymbol{t}}_{n}, \boldsymbol{v}\right\rangle_{\Gamma_{h}} \\
& +(\boldsymbol{u}, \nabla q)_{\Omega_{h}}-\langle\widehat{\boldsymbol{u}} \cdot \boldsymbol{n}, q\rangle_{\Gamma_{h}} \\
+(\boldsymbol{\sigma}, \boldsymbol{\tau})_{\Omega_{h}} & +(\boldsymbol{u}, \nabla \cdot \boldsymbol{\tau})_{\Omega_{h}}-\langle\widehat{\boldsymbol{u}}, \boldsymbol{\tau} \boldsymbol{n}\rangle_{\Gamma_{h}}=(\boldsymbol{f}, \boldsymbol{v})_{\Omega_{h}}=l(v) .
\end{aligned}
$$

Stokes Discretization. DPG does not impose any particular constraints on the basis functions used to represent the various components of the discrete solution. However, we do have the guarantee that the error in the solution is minimized in the energy norm, and under certain modest assumptions (see [48]) if we choose the graph norm as above, we have an inequality of the form

$$
\begin{aligned}
& \left(\left\|u-u_{h}\right\|^{2}+\left\|\widehat{u}-\widehat{u}_{h}\right\|_{\hat{H}_{A}\left(\Gamma_{h}\right)}^{2}\right)^{1 / 2} \\
& \leq \frac{M}{\gamma_{D P G}} \inf _{\left(w_{h}, \widehat{w}_{h}\right)}\left(\left\|u-w_{h}\right\|^{2}+\left\|\widehat{u}-\widehat{w}_{h}\right\|_{\hat{H}_{A}\left(\Gamma_{h}\right)}^{2}\right)^{1 / 2},
\end{aligned}
$$

where the group variables $u$ and $\widehat{u}$, defined above, are the exact solution, while $u_{h}$ and $\widehat{u}_{h}$ are their discrete solution counterparts, $M$ and $\gamma_{\mathrm{DPG}}$ are mesh-independent constants, and $\|\cdot\|_{\hat{H}_{A}\left(\Gamma_{h}\right)}$ is the "natural" norm on the traces, the minimum energy extension norm.

Assuming $u$ is sufficiently smooth, for a discrete $L^{2}$ space comprised of polynomials of order $k$, we expect best $h$-convergence rates of $k+1$; that is, we have

$$
\inf _{w_{h} \in U_{h}}\left\|u-w_{h}\right\| \leq C_{1} h^{k+1}
$$

for some mesh-independent constant $C_{1}$. It can be shown that, for traces $\widehat{w}_{h}$ whose $H^{-1 / 2}\left(\Gamma_{h}\right)$ and $H^{1 / 2}\left(\Gamma_{h}\right)$ components are approximated by polynomials of orders $k$ and $k+1$, respectively,

$$
\inf _{\widehat{w}_{h} \in \hat{H}_{A}\left(\Gamma_{h}\right)}\left\|\widehat{u}-\widehat{w}_{h}\right\|_{\hat{H}_{A}\left(\Gamma_{h}\right)} \leq C_{2} h^{k+1}
$$

for some mesh-independent constant $C_{2}$. For details and further references, see [19, pp. 7-8]. Combining this with equations (2.5) and (2.6), we then have the bound

$$
\left\|u-u_{h}\right\| \leq C h^{k+1}
$$

for $C=\min \left(C_{1}, C_{2}\right)$.

We can also motivate the choice of polynomial orders for the trial space intuitively from the exact sequence. If we define $k$ as the polynomial order of approximation of field variables, because these belong to $L^{2}$, it is natural to 
choose $k+1$ as the $H^{1}$ order. The traces of $H^{1}(K)$ functions $\left(\widehat{u}_{1}\right.$ and $\left.\widehat{u}_{2}\right)$ belong to $H^{1 / 2}(\partial K)$, a stronger space than $L^{2}(K)$, so that $k+1$ is a natural order of approximation for these. The traces of $H(\operatorname{div}, \mathrm{K})$ functions $\widehat{\boldsymbol{t}}_{n}$ belong to $H^{-1 / 2}(\partial K)$, a weaker space than $L^{2}$, so that $k$ is a natural order of approximation for these.

Thus, the natural choice for our trial space discretization is a polynomial space such that

$$
\begin{aligned}
& \boldsymbol{u} \in \mathbf{P}^{k}(K), \widehat{\boldsymbol{u}} \in \mathbf{P}^{k+1}(\partial K), \\
& p \in \mathbf{P}^{k}(K), \\
& \boldsymbol{\sigma} \in \mathbf{P}^{k}(K), \widehat{\boldsymbol{t}}_{n} \in \mathbf{P}^{k}(\partial K),
\end{aligned}
$$

for each element $K$. With this choice of space, we can expect our discrete solution to converge at a rate of $k+1$, provided that the exact solution is smooth.

Note that the fact that $\widehat{\boldsymbol{u}} \in H^{1 / 2}\left(\Gamma_{h}\right)$ suggests that we should enforce continuity at vertices; while the fact that $\widehat{\boldsymbol{t}}_{n} \in H^{-1 / 2}\left(\Gamma_{h}\right)$ suggests that we should allow discontinuities at the vertices.

Finally, we must choose a discretization for our test space. Again we have considerable freedom, but a natural choice is an element-wise basis that conforms to spaces supporting the differential operators taken on the test space. That is, since we take the divergence of $\boldsymbol{\tau}$, a natural choice for $\boldsymbol{\tau}$ is a vector $H$ (div)-conforming basis. In terms of polynomial order, we take the maximum $k+1$ order of discretization used in our trial space, and "enrich" it by some amount $\Delta k$. In the present work, we use $\Delta k=2$. The essential tradeoff is between local computational costs (the choice of test space polynomial order only affects the determination of optimal test functions, a local operation) and accurate determination of the optimal test functions.

Thus we select a test space such that

$$
\begin{aligned}
& \boldsymbol{v} \in \mathbf{P}^{k+1+\Delta k}(K) \subset \boldsymbol{H}^{1}(K), \\
& q \in \mathbf{P}^{k+1+\Delta k}(K) \subset H^{1}(K), \\
& \boldsymbol{\tau} \in \mathbf{P}^{k+1+\Delta k}(K) \subset \boldsymbol{H}(\operatorname{div}, \mathrm{K}) .
\end{aligned}
$$

In the present work, we employ nodal conforming bases provided by Trilinos's Intrepid package.

Stokes Boundary Conditions. As indicated above, we take our field variables-that is, $\boldsymbol{u}, p$, and $\boldsymbol{\sigma}$ - to be in $L^{2}$. We therefore replace the boundary condition $\boldsymbol{u}=\boldsymbol{g}$ on $\partial \Omega_{h}$ with the condition $\widehat{\boldsymbol{u}}=\boldsymbol{g}$ on $\partial \Omega_{h}$. We discuss imposition of the zero-mean condition on the pressure below, in the context of stiffness matrix assembly.

Stokes Test Space Inner Product. The test space inner product is a crucial choice in DPG; it determines the energy norm, in which the method is optimal. Suppose we want a method optimal in the $L^{2}$ norm of our field variables; that is, we wish to minimize

$$
\left\|\boldsymbol{u}-\boldsymbol{u}_{h}\right\|^{2}+\left\|\boldsymbol{\sigma}-\boldsymbol{\sigma}_{h}\right\|^{2}+\left\|p-p_{h}\right\|^{2}
$$

As we have previously discussed in some depth [48], an appropriate choice for the test norm in this case is the graph norm. Because our focus in the present discussion is on execution, here we limit ourselves to the determination of the 
graph norm. Grouping equation (2.4) by the field variables, we have

$$
\begin{aligned}
= & (\boldsymbol{u}, \nabla q+\nabla \cdot \boldsymbol{\tau})_{\Omega_{h}}+(p, \nabla \cdot \boldsymbol{v})_{\Omega_{h}}+(\boldsymbol{\sigma}, \nabla \boldsymbol{v}+\boldsymbol{\tau})_{\Omega_{h}} \\
& +\langle\text { boundary terms }\rangle
\end{aligned}
$$

The graph norm is then given by the Euclidean combination of the test terms for each field variable, plus $L^{2}$ norms ${ }^{3}$ of each test variable:

$$
\|(\boldsymbol{v}, q, \boldsymbol{\tau})\|_{\text {graph }}^{2}=\|\nabla q+\nabla \cdot \boldsymbol{\tau}\|^{2}+\|\nabla \cdot \boldsymbol{v}\|^{2}+\|\nabla \boldsymbol{v}+\boldsymbol{\tau}\|^{2}+\|\boldsymbol{v}\|^{2}+\|q\|^{2}+\|\boldsymbol{\tau}\|^{2}
$$

Optimal Test Function Determination. As discussed above, each trial space basis function $e_{i}$ has corresponding to it an optimal test function $v_{e_{i}}$, where $v_{e_{i}}$ solves the equation

$$
\left(v_{e_{i}}, \delta v\right)_{V}=b\left(e_{i}, \delta v\right) \quad \forall \delta v \in V
$$

where $V$ is the (discrete) test space. Because we employ test functions that are allowed to be discontinuous at interelement boundaries, this is an element-local problem. Note that because of the discretizations we have selected, the test space will have more degrees of freedom - if we take the test space to have $m$ degrees of freedom per element and the trial space to have $n$ degrees of freedom, then the left hand side of this system will be a square $m \times m$ matrix, and the right a rectangular $m \times n$ matrix (expanding the equation column-wise in the trial space index $i$ ), and the solution will be $n$ vectors of length $m$ : the $m$ coefficients of each optimal test function. If our basis for the enriched test space consists of functions $v_{j}$ and we define the $m \times m$ Gram matrix

$$
G_{j k}=\left(v_{j}, v_{k}\right)_{V}
$$

and the $n \times m$ bilinear form matrix

$$
B_{i j}=b\left(e_{i}, v_{j}\right)
$$

then the optimal test coefficients are the columns of $G^{-1} B^{T}$.

Stiffness Matrix Assembly. Once the optimal test functions for an element are computed, the element stiffness matrix $K_{i j}$ is relatively simple to compute; we have

$$
K_{i j}=b\left(e_{i}, v_{e_{j}}\right)=\left(v_{e_{i}}, v_{e_{j}}\right)_{V}
$$

by the very problem that we solved to determine the optimal test functions. Note that we can compute the inner product of an arbitrary pair of test basis functions $\alpha_{i} v_{i}$ and $\beta_{j} v_{j}$ by means of the Gram matrix:

$$
\left(\alpha_{i} v_{i}, \beta_{j} v_{j}\right)_{V}=\boldsymbol{\alpha}^{T}\left(v_{i}, v_{j}\right)_{V} \boldsymbol{\beta}=\boldsymbol{\alpha}^{T} G \boldsymbol{\beta},
$$

\footnotetext{
${ }^{3}$ The $L^{2}$ terms may be weighted by constants; the best choice of constants will depend on the problem. For the present work, we have simply taken unit weights.
} 
so that the element stiffness matrix is given by

$$
\left(v_{e_{i}}, v_{e_{j}}\right)_{V}=\left(G^{-1} B^{T}\right)^{T} G G^{-1} B^{T}=B G^{-1} B^{T}
$$

The element stiffness matrix entries can then be assembled into a global stiffness matrix. Note that the only interelement coupling comes through the abstract trace variables (the trace $\widehat{\boldsymbol{u}}$ and flux $\widehat{\boldsymbol{t}}_{n}$, in the case of Stokes).

To impose the zero-mean condition on the pressure, we follow a technique described by Bochev and Lehoucq [2], which preserves symmetric positive definiteness of the stiffness matrix and allows a simple implementation for nodal bases (which is what we use for field variables in Camellia).

Global Problem Solution. As noted above, the only entries in our stiffness matrix that are coupled between elements belong to trace variables. We can therefore employ static condensation to reduce the size of the global system. That is, the matrix can be reordered to take the form

$$
\left(\begin{array}{ll}
K_{11} & K_{12} \\
K_{12}^{T} & K_{22}
\end{array}\right)\left(\begin{array}{c}
u \\
f
\end{array}\right)=\left(\begin{array}{l}
F_{1} \\
F_{2}
\end{array}\right)
$$

where $K_{11}$ is block diagonal, $u$ represents the degrees of freedom corresponding to field variables, and $f$ those corresponding to the trace variables. Noting that $u=K_{11}^{-1}\left(F_{1}-K_{12} f\right)$, we can substitute this into the equation $K_{12}^{T} u+K_{22} f=F_{2}$ to obtain an equation for the trace degrees of freedom:

$$
\left(K_{22}-K_{12}^{T} K_{11}^{-1} K_{12}\right) f=F_{2}-K_{12}^{T} K_{11}^{-1} F_{1}
$$

Since $K_{11}$ is block diagonal, its inversion can be carried out element-wise and in parallel; since $K_{12}$ is a significantly smaller matrix, the computational cost of the global solve is reduced. For simplicity, we have generally employed direct solvers for both the local and the global solves.

\subsection{Riesz Representations and Adaptivity}

DPG minimizes the error in the energy norm; that is, for a problem with exact solution $u$ and discrete solution $u_{h}$,

$$
\left\|u-u_{h}\right\|_{E}=\sup _{\|v\|_{V}=1} b\left(u-u_{h}, v\right)
$$

is minimized. Now, we may rewrite this as

$$
\begin{aligned}
\sup _{\|v\|_{V}=1} b\left(u-u_{h}, v\right) & =\sup _{\|v\|_{V}=1}\left(b(u, v)-b\left(u_{h}, v\right)\right) \\
& =\sup _{\|v\|_{V}=1}\left(l(v)-b\left(u_{h}, v\right)\right) \\
& =\left\|l-B u_{h}\right\|_{V^{\prime}},
\end{aligned}
$$

where the operator $B$ is defined by $B u=b(u, \cdot)$. The error $\left\|l-B u_{h}\right\|_{V^{\prime}}$ is computable by virtue of the Riesz representation theorem, which tells us that there exists a function $e \in V$ such that

$$
(e, v)_{V}=l(v)-b\left(u_{h}, v\right) \quad \forall v \in V
$$


and moreover that $\|e\|_{V}=\left\|l-B u_{h}\right\|_{V^{\prime}}$. We call this $e$ the error representation function, and compute it by defining a residual vector

$$
r_{i}=l\left(v_{i}\right)-b\left(u_{h}, v_{i}\right)
$$

then inverting the Gram matrix to solve for $e=G^{-1} r$. We can then compute

$$
\|e\|_{V}^{2}=(e, e)_{V}=e^{T} G e=\left(G^{-1} r\right)^{T} G e=r^{T} e .
$$

Note that this computation is local to the element, making it susceptible to parallel execution. Once we have computed the error, any number of refinement strategies might be employed. Our basic strategy is a greedy one, determining the maximum element error $\left\|e_{K}\right\|_{V}$ in the mesh, and marking for refinement any element with error greater than $\theta\left\|e_{K}\right\|_{V}$, where $\theta \in(0,1)$ is a threshold parameter, which in most of the computations here presented we have taken to be 0.20 . The refinements might be either $h$ - or $p$-refinements.

When one element is more refined than its neighbor (in either $h$ or $p$ ), we must decide what discretization to use for the trace variables along the interface between the elements. While other choices are possible, our usual approach is to adopt the finer element's discretization along the interface.

\subsection{Navier-Stokes Formulation}

DPG minimizes the residual of a linear problem. While more sophisticated approaches are possible, for the present when solving a nonlinear problem we first linearize the problem, then use DPG to solve the linearized problem. For example, we may write the steady Navier-Stokes equations as:

$$
\begin{aligned}
-\nabla p+\mu \nabla \cdot \boldsymbol{\sigma} & =\boldsymbol{f}+\boldsymbol{u} \cdot \nabla \boldsymbol{u} \\
\boldsymbol{\sigma}-\nabla \boldsymbol{u} & =0 \\
\nabla \cdot \boldsymbol{u} & =0
\end{aligned}
$$

Observing that the nonlinear, convective term may be written $\boldsymbol{u} \cdot \nabla \boldsymbol{u}=\boldsymbol{u} \cdot \boldsymbol{\sigma}$, we immediately see that a Navier-Stokes formulation corresponding to our VGP Stokes formulation is

$$
\begin{aligned}
\left\langle\widehat{\boldsymbol{t}}_{\boldsymbol{n}}, \boldsymbol{v}\right\rangle_{\Gamma_{h}}+(p, \nabla \cdot \boldsymbol{v})_{\Omega_{h}}+(\boldsymbol{\sigma}, \nabla(\mu \boldsymbol{v}))_{\Omega_{h}}-(\boldsymbol{u} \cdot \boldsymbol{\sigma}, \boldsymbol{v})_{\Omega_{h}} & =(\boldsymbol{f}, \boldsymbol{v})_{\Omega_{h}}, \\
(\boldsymbol{\sigma}, \boldsymbol{\tau})_{\Omega_{h}}-\langle\widehat{\boldsymbol{u}}, \boldsymbol{\tau} \boldsymbol{n}\rangle_{\Gamma_{h}}+(\boldsymbol{u}, \nabla \cdot \boldsymbol{\tau})_{\Omega_{h}} & =0 \\
\langle\widehat{\boldsymbol{u}} \cdot \boldsymbol{n}, q\rangle_{\Gamma_{h}}-(\boldsymbol{u}, \nabla q)_{\Omega_{h}} & =0 .
\end{aligned}
$$

If we define the Stokes bilinear formulation as $b_{\text {Stokes }}(u, v)=l_{\text {Stokes }}(v)$, and linearize about $(\boldsymbol{u}+\Delta \boldsymbol{u}, \boldsymbol{\sigma}+\Delta \boldsymbol{\sigma}, p+$ $\Delta p$ ), we then have

$$
b_{\text {Stokes }}(\Delta u, v)-(\Delta \boldsymbol{u} \cdot \boldsymbol{\sigma}+\boldsymbol{u} \cdot \Delta \boldsymbol{\sigma}, \boldsymbol{v})_{\Omega_{h}}=(\boldsymbol{f}, \boldsymbol{v})_{\Omega_{h}}-b_{\text {Stokes }}(u, v)+(\boldsymbol{u} \cdot \boldsymbol{\sigma}, \boldsymbol{v})_{\Omega_{h}} .
$$

Our strategy for solving this is a standard Newton iteration: we start from some initial guess $u=u_{0}$, solve for the increment $\Delta u$, set $u_{i+1}=u_{i}+\Delta u$, and iterate until some measure of the increment is below a desired threshold. It is worth noting, however, that in general our test norm will now depend on the background flow ${ }^{4} u_{i}$.

\footnotetext{
${ }^{4}$ That is, the previous solution.
} 


\subsubsection{Stopping Criterion for Nonlinear Iteration}

Our stopping criterion for the Newton-Raphson iteration in the context of adaptivity is as follows. We define an $L^{2}$ tolerance $\epsilon_{0}$ for the initial mesh (that is, refinement 0 ). After we converge sufficiently that the $L^{2}$ norm of the field variables in the Newton-Raphson update is below $\epsilon_{0}$, we measure the energy norm of the solution, as well as the energy norm of the error, to determine a relative energy error $e_{\text {rel }}^{0}$ for refinement $i=0$, defined as:

$$
e_{\mathrm{rel}}^{i}=\frac{\left\|e^{i}\right\|_{E}}{\left\|u_{h}^{i}\right\|_{E}}=\frac{\left\|u-u_{h}^{i}\right\|_{E}}{\left\|u_{h}^{i}\right\|_{E}}=\frac{\left\|l-B u_{h}^{i}\right\|_{V^{\prime}}}{\left\|u_{h}^{i}\right\|_{E}} .
$$

We then refine according to the greedy refinement strategy described above, and for refinement $i>0$ we set the $L^{2}$ tolerance for our stopping criterion as

$$
\epsilon_{i}=e_{\mathrm{rel}}^{i-1} \epsilon_{0}
$$

The computed error on the adaptive mesh thus automatically guides the $L^{2}$ convergence that we attempt to achieve in the Newton-Raphson iteration.

\subsection{Curvilinear Meshes}

When using curved geometries, the order of finite element solution convergence is generally limited by the order of approximation of the geometry. Thus to achieve higher-order convergence we require higher-order geometry representations. We choose to employ an isoparametric representation of the geometry, in which the basis functions used in finite element computations are also used to represent the geometry. Compared with representing the geometry exactly in our computations, this is an appealing choice for two reasons: first, the isoparametric representation will in general be cheaper to compute; second, isoparametric geometry allows the exact representation of linearized rigid-body motion, a fact of engineering interest. Our approach here essentially follows Demkowicz et al. [17, pp. 195-210].

Consider a connected domain $\Omega \subset \mathbb{R}^{2}$. We assume that the domain can be partitioned into curvilinear triangles and quadrilaterals. An example of a domain with such a partition is shown in Figure 1. In order to work with such geometries, we must determine a systematic mechanism for constructing maps $\boldsymbol{x}(\boldsymbol{t})$ from reference elements to physical space.

Now, typically an engineer or analyst will not have ready to hand a representation of the interior of the domain; rather, there will be some description of the boundary of the domain. In what follows we assume that this description is parametrically defined. This assumption does impose some constraints-in some cases, geometry will be known implicitly, perhaps defined by the intersection of two surfaces. For further details on implicitly defined geometry, see [17, pp. 198-199] and [22, pp. 96-97].

We proceed as follows. Since we assume a parametrization of the domain boundary, it is natural further to assume a parametrization of the edges (interior edges might simply be taken to be straight lines). From the edge parametrizations it is possible to construct a transfinite interpolant [27], a mapping from a reference quadrilateral or triangle onto its curvilinear counterpart which is exact on the edges. The transfinite interpolant in hand-a representation of the exact geometry-we construct a projection-based interpolant, which approximates the geometry using precisely the polynomial basis employed in our finite element discretization. 
Suppose that for an element $K$, for each edge $e_{i} \subset \partial K$ connecting vertices $\boldsymbol{v}_{i}$ and $\boldsymbol{v}_{i+1}$ there is some given parametrization $\boldsymbol{x}_{i}:[0,1] \rightarrow e_{i}$ onto the edge such that $\boldsymbol{x}_{i}(0)=\boldsymbol{v}_{i}$ and $\boldsymbol{x}_{i}(1)=\boldsymbol{v}_{i+1}$. (We implicitly use modular arithmetic in vertex here and below — thus on a quadrilateral vertex 0 is identified with vertex 4 , and similarly 0 and 3 are identified on the triangle.) The vertex and edge numbering for the reference quadrilateral are shown in Figure 2. We now construct transfinite interpolants for quadrilateral elements; an analogous construction exists for triangles.

Transfinite Interpolation on the Quadrilateral. We define the edge bubble functions ${ }^{5} \Delta \boldsymbol{x}_{i}$ by subtracting the vertex contributions from the edge parametrizations in an appropriately blended fashion:

$$
\Delta \boldsymbol{x}_{i} \stackrel{\text { def }}{=} \boldsymbol{x}_{i}-(t-1) \boldsymbol{v}_{i}-t \boldsymbol{v}_{i+1}
$$

We define a bilinear interpolant $\boldsymbol{b}\left(t_{1}, t_{2}\right)$ determined by the vertices:

$$
\boldsymbol{b}\left(t_{1}, t_{2}\right) \stackrel{\text { def }}{=}\left(1-t_{1}\right)\left(1-t_{2}\right) \boldsymbol{v}_{0}+t_{1}\left(1-t_{2}\right) \boldsymbol{v}_{1}+t_{1} t_{2} \boldsymbol{v}_{2}+\left(1-t_{1}\right) t_{2} \boldsymbol{v}_{3}
$$

The transfinite interpolant is then given by

$$
\begin{aligned}
\boldsymbol{x}\left(t_{1}, t_{2}\right) \stackrel{\text { def }}{=} \boldsymbol{b}\left(t_{1}, t_{2}\right) & +\left(1-t_{2}\right) \Delta x_{0}\left(t_{1}\right)+t_{2} \Delta x_{2}\left(t_{1}\right) \\
& +\left(1-t_{1}\right) \Delta x_{3}\left(t_{2}\right)+t_{1} \Delta x_{1}\left(t_{2}\right) .
\end{aligned}
$$

It is clear from the construction that this agrees exactly with the curves $\boldsymbol{x}_{i}$ on the element boundary, and that it blends the $\boldsymbol{x}_{i}$ in a continuous fashion on the element interior.

\footnotetext{
${ }^{5} \mathrm{~A}$ bubble function on a topological entity is defined to be a function that vanishes on that entity's boundary. Thus the edge bubbles vanish at the vertices, face bubbles vanish on edges, and so on.
}

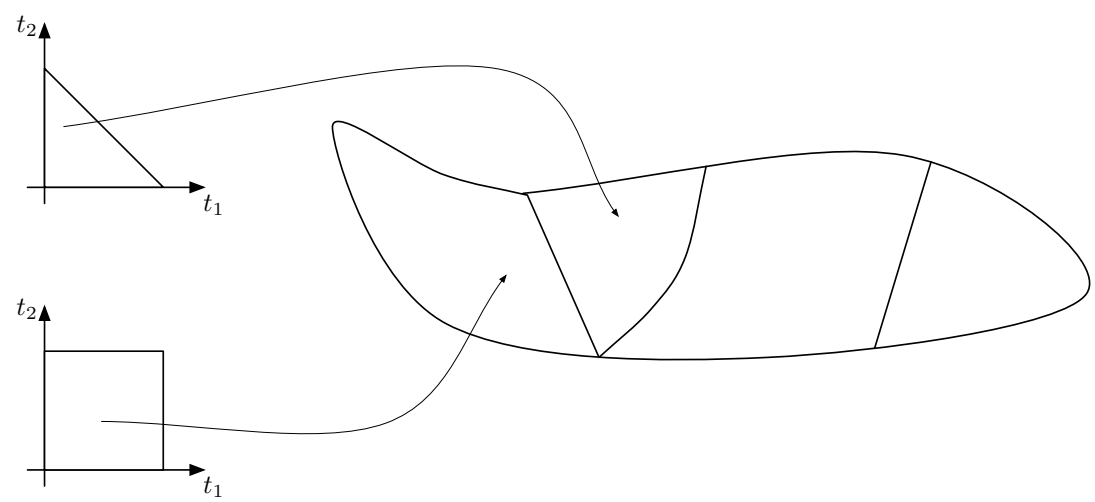

Figure 1: An example curvilinear domain partitioned into triangular and quadrilateral elements.

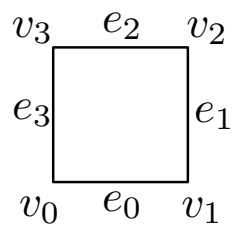

Figure 2: Vertex and edge numbering on reference quadrilateral. 
$\boldsymbol{H}^{1}$ projection-based interpolation. Thus far, our representation of the geometry has been exact. For reasons suggested above, we would like to determine a polynomial approximation to the geometry which will also allow us to maintain convergence rates. The correct way to do so is known as projection-based interpolation [17, pp. 180-183]. In our finite element discretization, it is desirable that elements should be compatible in the sense that they agree exactly on the location of shared vertices (and vertices on the approximate boundary should coincide with the exact boundary) as well as on the approximation of shared edges. Further, we would like our approximate geometry to closely approximate both location (that is, value) and curvature (derivative) information. We thus arrive at a constrained projection problem, which we can divide into steps:

1. Interpolate the vertices (i.e. use the vertex locations to set weights for the vertex basis functions).

2. Project the exact transfinite interpolant bubble - that is, $\Delta \boldsymbol{x} \stackrel{\text { def }}{=} \boldsymbol{x}\left(t_{1}, t_{2}\right)-\boldsymbol{b}\left(t_{1}, t_{2}\right)$-into the discrete space of edge bubble functions. Call the sum of the weighted edge and vertex functions $\widetilde{\boldsymbol{x}}_{\text {edge. }}$.

3. Project the difference of the function thus far and the transfinite interpolant - that is, $\Delta \boldsymbol{x}-\widetilde{\boldsymbol{x}}_{\text {edge }}-$ into the discrete space of face bubble functions.

Refinements. It remains to specify how refinements should be handled: specifically, should the edges interior to the refined element be curves defined as above by the transfinite interpolant, or will straight edges suffice? For standard conforming elements, the usual practice is to use curved edges on the interior to guarantee optimal convergence rates. We were unsure whether this would be necessary for DPG in the curvilinear geometry of immediate interest to us, so we simply experimented with using straight edges on element interiors- the computational advantage being that we reduce the number of elements for which we need to compute the curvilinear geometry, and the refinements are somewhat simpler to implement this way. It is worth noting that we do use the transfinite interpolant to compute the location of the new vertices. We have verified the approach and the code using a Poisson manufactured solution on a domain with an embedded circle, for which our solution matches the best approximation in the space. This is not a proof, and we do hope in the future to implement refinements that are curvilinear on their interiors so that we can study the relative costs and benefits of the two approaches.

It is worth noting in this context that for certain meshes and geometries, clearly refinements with straight-edged interiors will not suffice - for example, if a sufficiently thin, sufficiently curved shell element is refined, the straight edges on the interior of a refinement will intersect the curved edges on the element's boundary. In the present work, we only employ one curvilinear geometry of modest curvature-flow past a cylinder.

\section{Numerical Experiments}

To assess the efficacy of our approach, we first examine a smooth manufactured solution due to Kovasznay [32] on uniform meshes. We then turn to three classical model problems: lid-driven cavity flow, flow past a backward-facing step, and flow past a cylinder. For each of these, we perform a series of experiments using automatic adaptivity based on the DPG error measurement. 


\subsection{Kovasznay Flow}

A common test case for Navier-Stokes is an analytic solution due to Kovasznay [32]:

$$
\begin{aligned}
u_{1} & =1-e^{\lambda x} \cos (2 \pi y) \\
u_{2} & =\frac{\lambda}{2 \pi} e^{\lambda x} \sin (2 \pi y) \\
p & =\frac{1}{2} e^{2 \lambda x}+C
\end{aligned}
$$

where $\lambda=\frac{\mathrm{Re}}{2}-\sqrt{\left(\frac{\mathrm{Re}}{2}\right)^{2}+(2 \pi)^{2}}$. We use $\Omega=(-0.5,1.5) \times(0,2)$ as our domain, and choose the constant $C$ so that $p$ has zero average on $\Omega$.

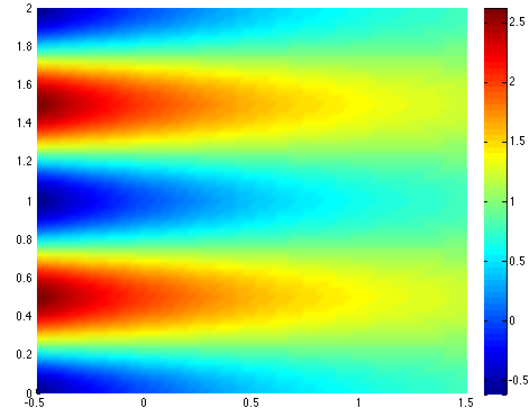

(a) $u_{1}$

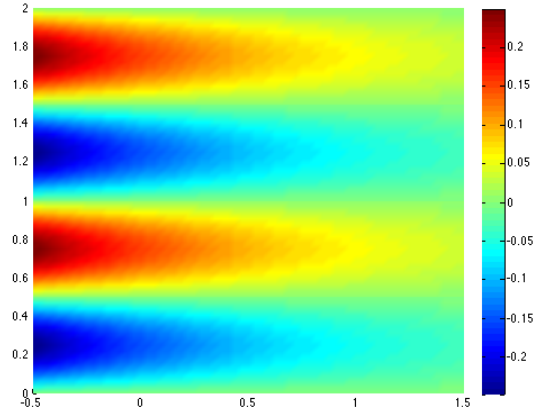

(b) $u_{2}$

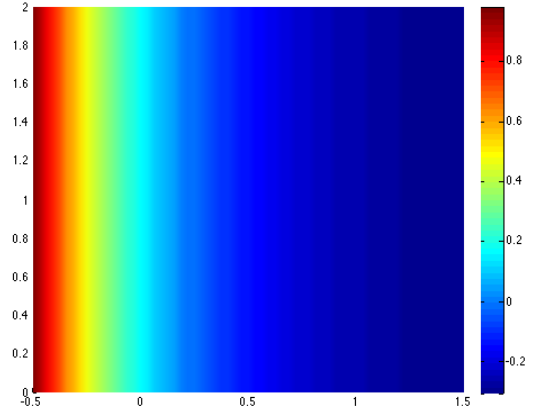

(c) $p$

Figure 3: Kovasznay flow for $\operatorname{Re}=40: u_{1}, u_{2}$ and $p$.

A standard test case for Kovasznay flow is $R e=40$; the solution for this case is plotted in Figure 3. For our first test, we use the standard graph norm and $k=1$ to 4 and $\Delta k=2$, on meshes from $1 \times 1$ to $64 \times 64$ elements. Figure 4 shows the velocity error in the DPG solution as well as the $L^{2}$-projection exact solution (the best approximation); the results are essentially perfect. The pressure results are shown in Figure 5-here, there is a gap between the best error and that achieved by DPG, but the two are generally close, and DPG converges at appropriate rates. Finally, 
the euclidean combination of all field variables is shown in Figure 6-since this is the norm we sought optimality in by using the graph norm, this is the "fairest" one to examine, and here the results are again essentially perfect. The explanation, then, for the sub-optimal pressure results is simply that the error in other components dominates.
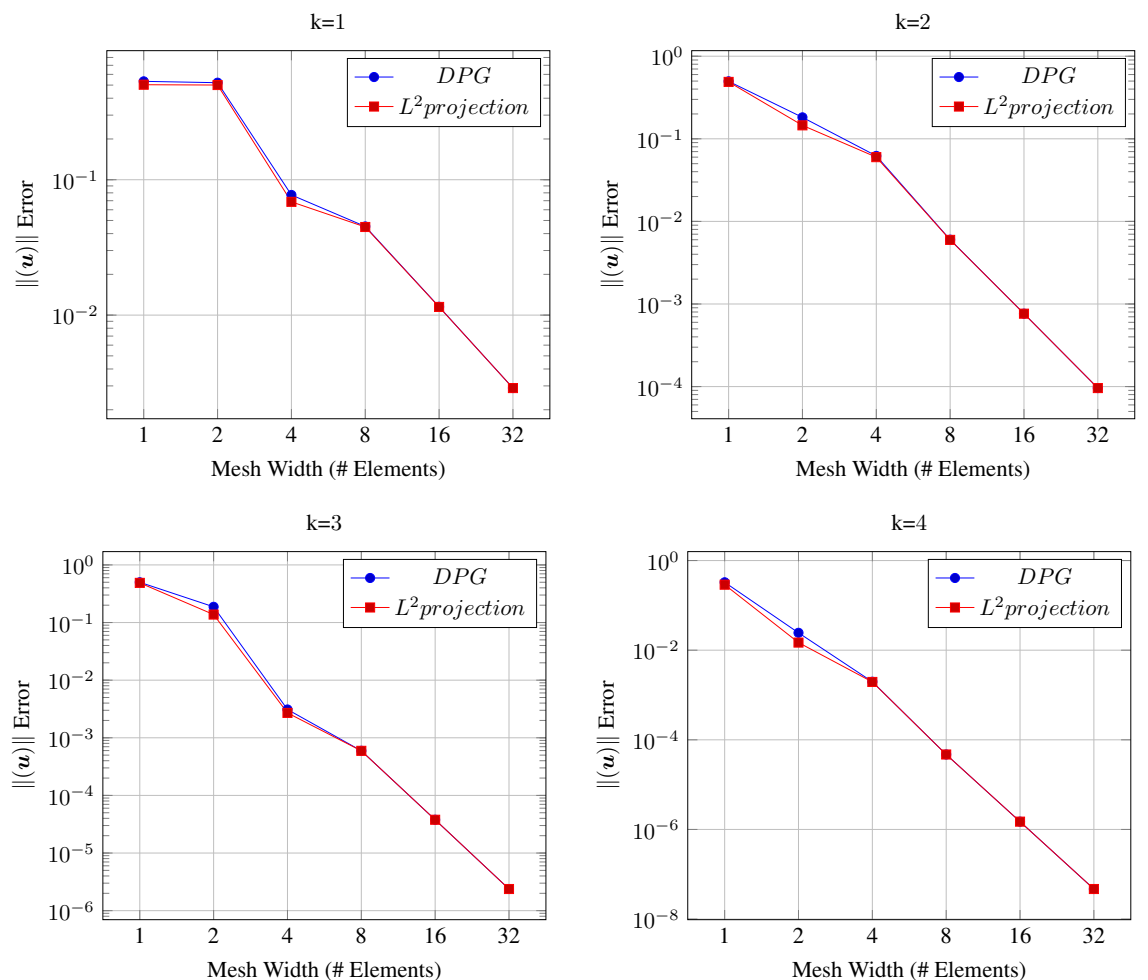

Figure 4: Kovasznay flow results for the DPG Navier-Stokes formulation using the standard graph norm with Re = 40: velocity error.

\subsection{Lid-Driven Cavity Flow}

A classic test case for incompressible flow is the lid-driven cavity flow problem. Consider a square cavity with an incompressible, viscous fluid, with a lid that moves at a constant rate. The resulting flow will be vorticular; as sketched in Figure 7, there will also be so-called Moffatt eddies at the corners; in fact, the exact solution will have an infinite number of such eddies, visible at progressively finer scales [37]. Note that the problem as described will have a discontinuity in the fluid velocity at the top corners, and hence its solution will not conform to the spaces we used in our analysis; for this reason, in our experiment we approximate the problem by introducing a thin ramp in the 

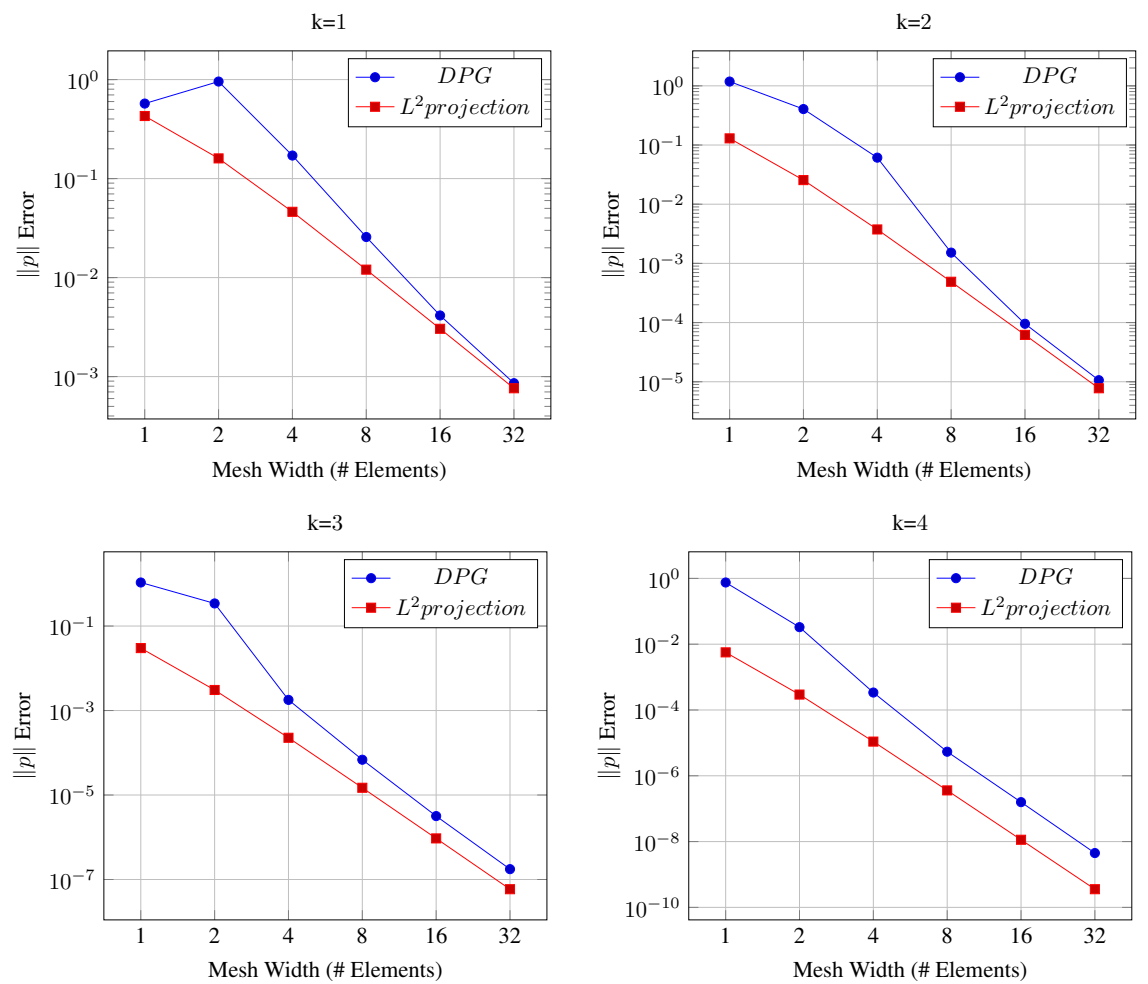

Figure 5: Kovasznay flow results for the DPG Navier-Stokes formulation using the standard graph norm with Re $=40$ : pressure error.

boundary conditions — we have chosen a ramp of width $\frac{1}{64}$. This makes the boundary conditions continuous, ${ }^{6}$ so that the solution conforms to the spaces used in the analysis. Here, we seek first of all to show that the method converges to solutions that qualitatively agree with accepted solutions in the literature; while we do present some careful (and encouraging) quantitative comparisons in the context of the $\mathrm{Re}=1000$ lid-driven cavity problem, such comparisons are not our principal objective.

Fixed Meshes. Starting with a zero initial guess, and using the VGP Navier-Stokes Formulation presented above with its adjoint graph norm, we solved the lid-driven cavity flow problem for $\operatorname{Re}=100,400$, and 1000. The resulting streamline plots are shown in Figure 8.

We then solve each of these flow regimes using Navier-Stokes with adaptivity. We use the energy error of the linearized problem to drive refinements according to our usual greedy strategy with a threshold $\theta=20 \%$. In each case, we start with a $2 \times 2$ quartic mesh and perform six refinement steps. On the coarsest mesh, we start from a zero initial guess; for subsequent meshes, we use the projection of the solution thus far onto the refined mesh. Since our solution is linear in the fluxes and traces, we do not accumulate in these, but instead solve for these at each Newton

\footnotetext{
${ }^{6}$ It is worth noting that these boundary conditions are not exactly representable by many of the coarser meshes used in our experiments. We interpolate the boundary conditions in the discrete space.
} 

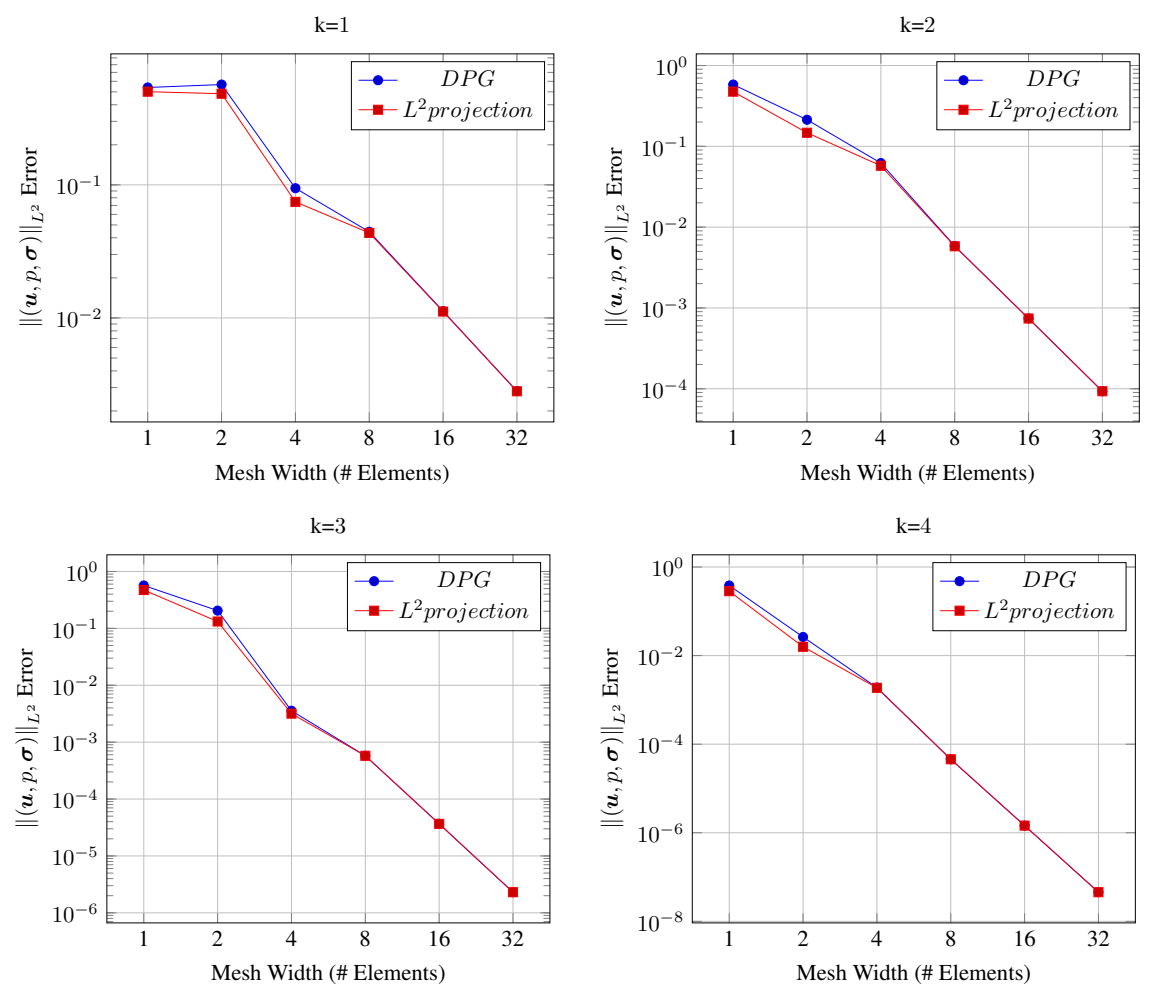

Figure 6: Kovasznay flow results for the DPG Navier-Stokes formulation using the standard graph norm with Re $=40$ : $L^{2}$ error of all field variables.

step. We continue Newton iterations on each refined mesh until the $L^{2}$ norm of the field variable increments-that is, $\left(\|\boldsymbol{u}\|^{2}+\|p\|^{2}+\|\boldsymbol{\sigma}\|^{2}\right)^{1 / 2}$ —was less than a tolerance of $3 \times 10^{-8}$. On the final solve, we required a tighter tolerance of $3 \times 10^{-9}$. $^{7}$ The resulting streamlines are shown in Figures 9, 10, and 11. The reported energy errors are for the linearized problem in the last Newton step.

For a Reynolds number of 5000, the strategy outlined above does not converge, presumably due to roundoff error in the solution of the optimal test functions or (more likely) in the solution of the global problem. To solve in this regime, we modified the strategy slightly: we used simple artificial time stepping with a fixed time step size of 1.0. Here, we relaxed the nonlinear tolerances to $10^{-3}$ for the intermediate solves, and $10^{-4}$ for the final solve. The results are shown in Figure 12.

For the $\operatorname{Re}=8000$ case, we used an identical approach to the one we used for $R e=5000$; the results are shown in 13. It is worth noting that somewhere between $R e=8000$ and $R e=8050$, the flow becomes oscillatory [8].

Despite the fact that the flow is known to become oscillatory for Reynolds numbers above 8050, we have found that we can compute solutions for higher Reynolds numbers using our steady-state solver. For example, Figure 14 shows

\footnotetext{
${ }^{7}$ Note that these simple stopping criteria differ from the dynamic nonlinear stopping criterion articulated in Section 2.4.1. We employ the dynamic criterion for the backward-facing step and flow past a cylinder experiments.
} 


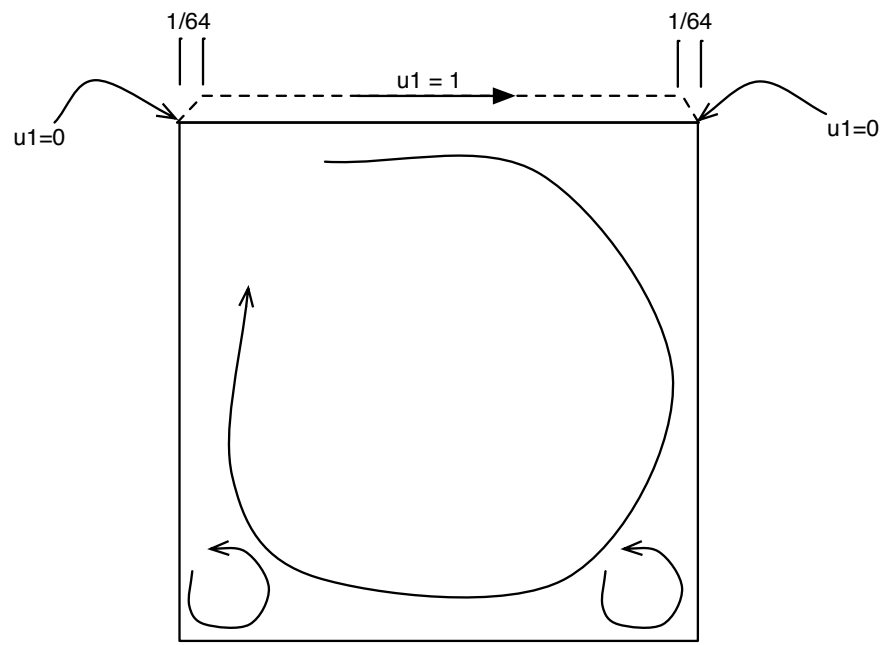

Figure 7: Sketch of lid-driven cavity flow. To obtain a solution in $H^{1}$, we linearly interpolate the lid boundary condition $u_{1}=1$ and the wall condition $u_{1}=0$ in segments of width $\frac{1}{64}$ on the left and right ends of the lid.

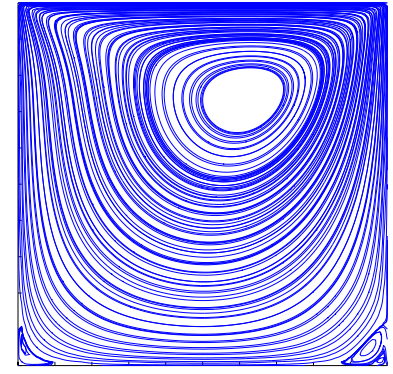

(a) $\operatorname{Re}=100$

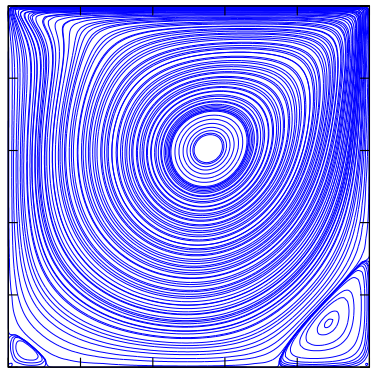

(b) $\operatorname{Re}=400$

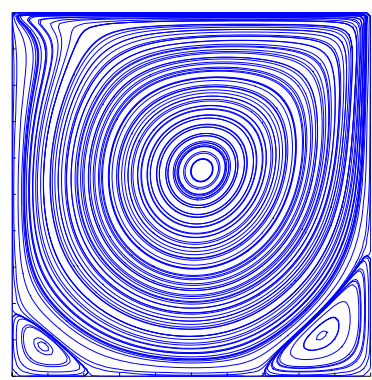

(c) $\operatorname{Re}=1000$

Figure 8: Streamlines for the cavity flow problem with $R e=100,400$, and 1000, each on a linear $64 \times 64$ mesh. We used $\Delta k=2$ for the test space enrichment. 


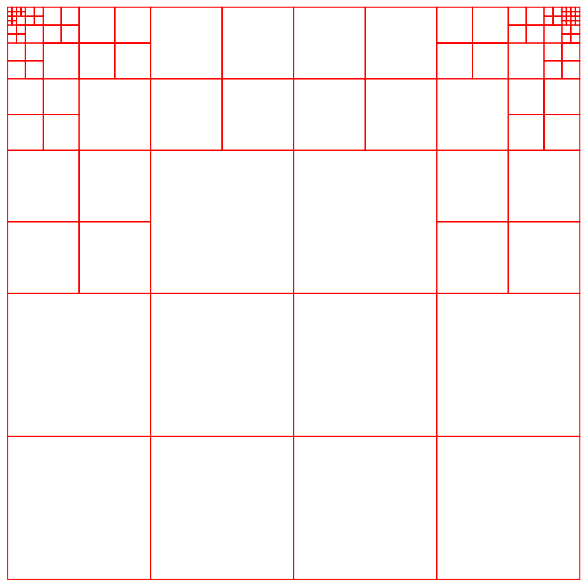

(a) mesh

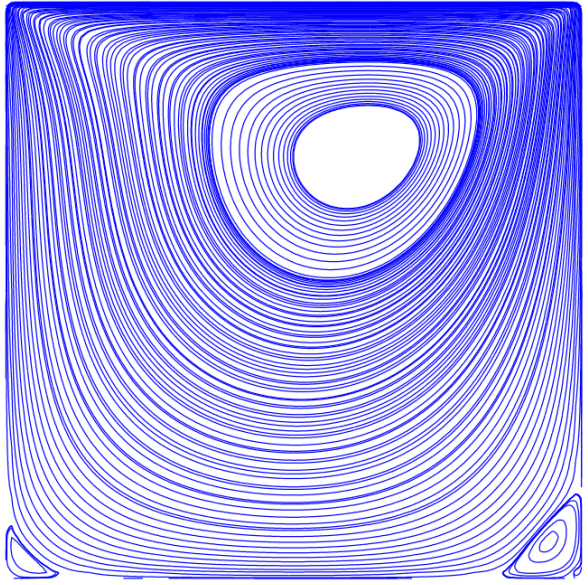

(b) streamlines

Figure 9: Streamlines for the cavity flow problem with $\mathrm{Re}=100$ on an adaptive quartic mesh after 6 refinements; final mesh has 109 elements $\left(24,159\right.$ dofs). The energy error of the solution is $2.7 \times 10^{-3}$.

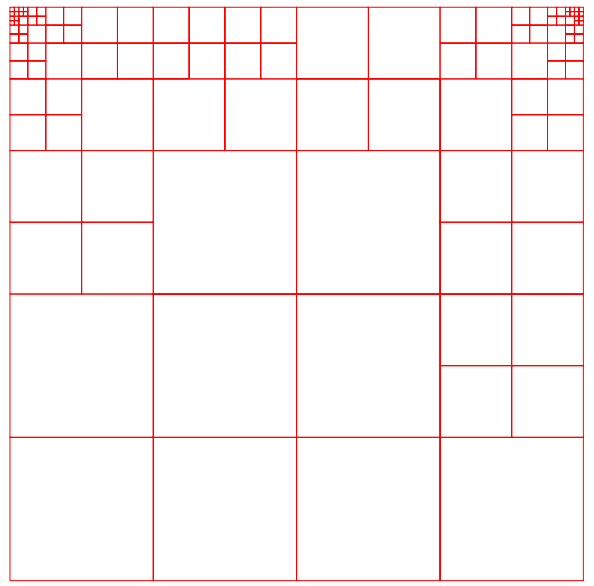

(a) mesh

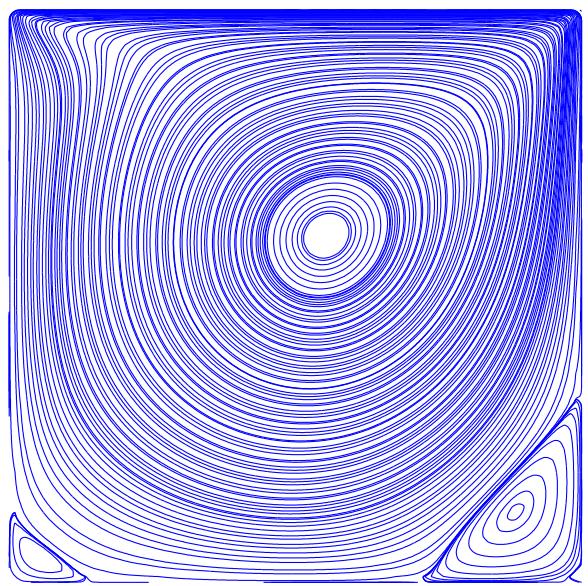

(b) streamlines

Figure 10: Streamlines for the cavity flow problem with $R e=400$ on an adaptive quartic mesh after 6 refinements; final mesh has 109 elements $\left(24,159\right.$ dofs). The energy error of the solution is $7.1 \times 10^{-4}$. 


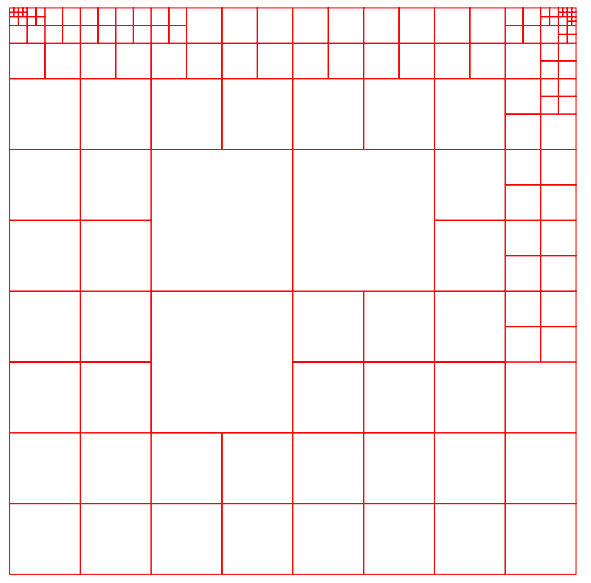

(a) mesh

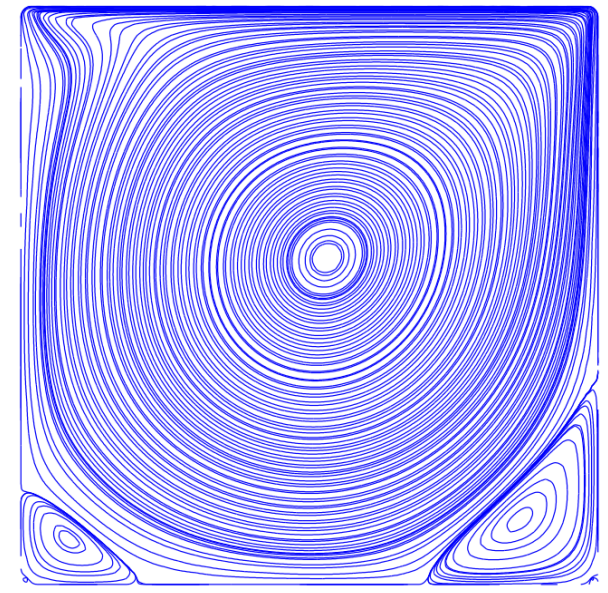

(b) streamlines

Figure 11: Streamlines for the cavity flow problem with $\mathrm{Re}=1000$ on an adaptive quartic mesh after 6 refinements; final mesh has 148 elements (32,686 dofs). The energy error of the solution is $2.9 \times 10^{-4}$.

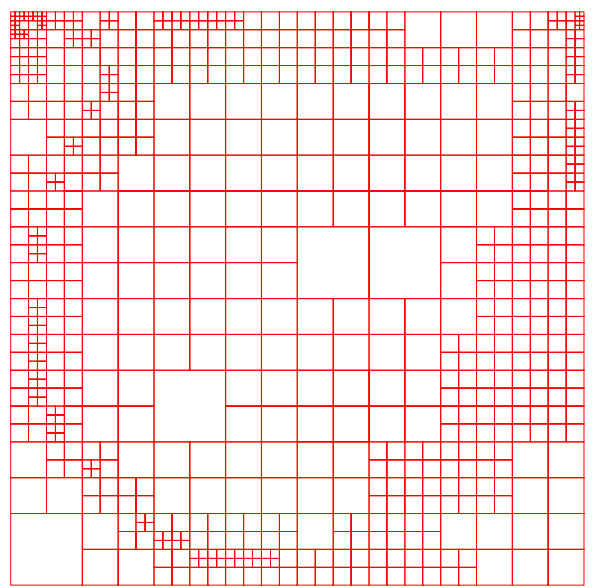

(a) mesh

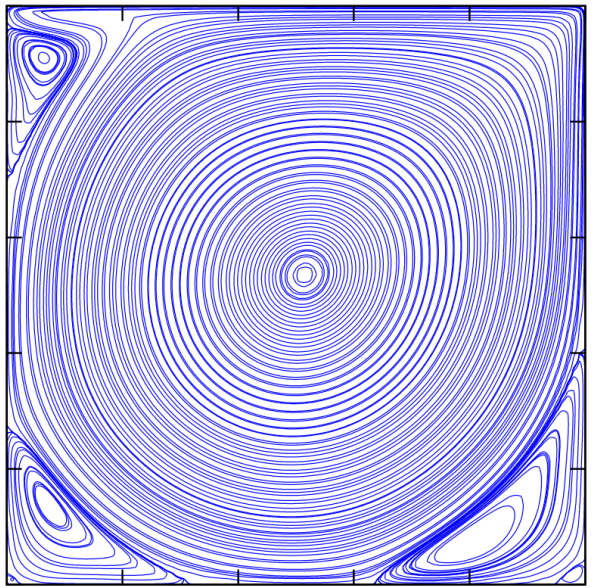

(b) streamlines

Figure 12: Streamlines for the cavity flow problem with $\mathrm{Re}=5000$ on an adaptive quartic mesh after 6 refinements and using artificial time stepping with $\mathrm{dt}=1.0$; final mesh has 763 elements $(166,001 \mathrm{dofs})$. The energy error of the solution is $1.6 \times 10^{-4}$. 
a solution with Reynolds number of 10000 , computed just as in the Re $=8000$ case. The streamlines appear quite similar to plots of the transient solution provided by Bruneau and Saad [8]. We are unsure of the best interpretation of this solution; perhaps it is an unstable steady state - the fact that we are able to resolve to an energy error of $3.0 \times 10^{-4}$ does seem to suggest that this is a solution to the problem.

For numerical verification of our cavity flow solutions, we compare our Re $=1000$ results with the extensive pseudo-spectral benchmark solution computed by Botella and Peyret [3]. We have computed many of the values

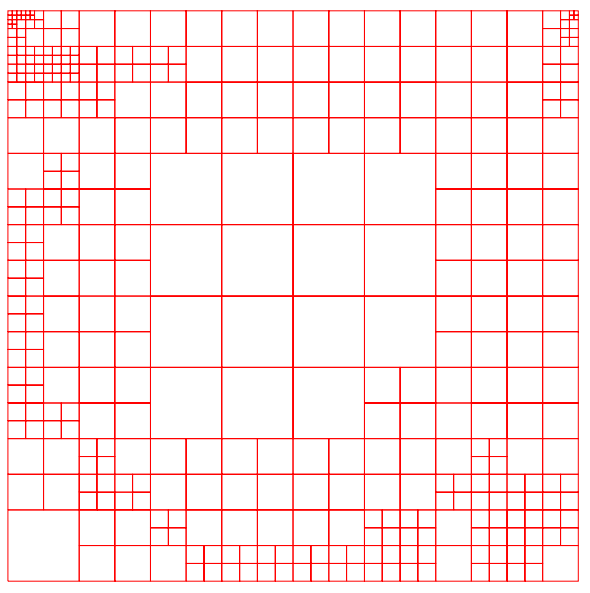

(a) mesh

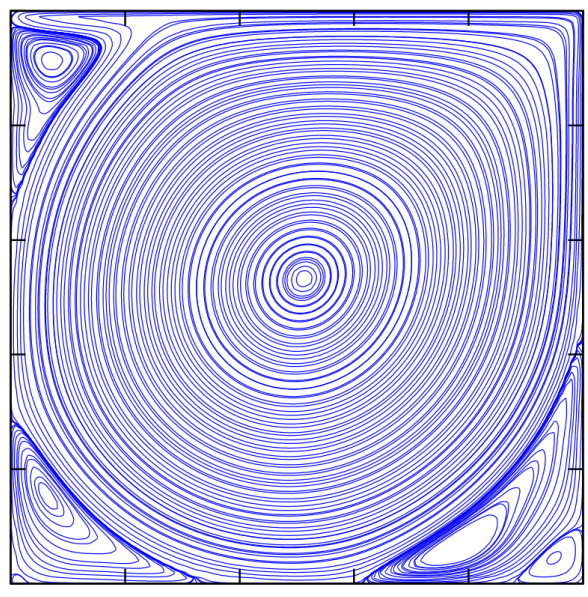

(b) streamlines

Figure 13: Streamlines for the cavity flow problem with $\mathrm{Re}=8000$ on an adaptive quartic mesh after 6 refinements and using artificial time stepping with $\mathrm{dt}=1.0$; final mesh has 400 elements (87,302 dofs). The energy error of the solution is $2.6 \times 10^{-4}$.

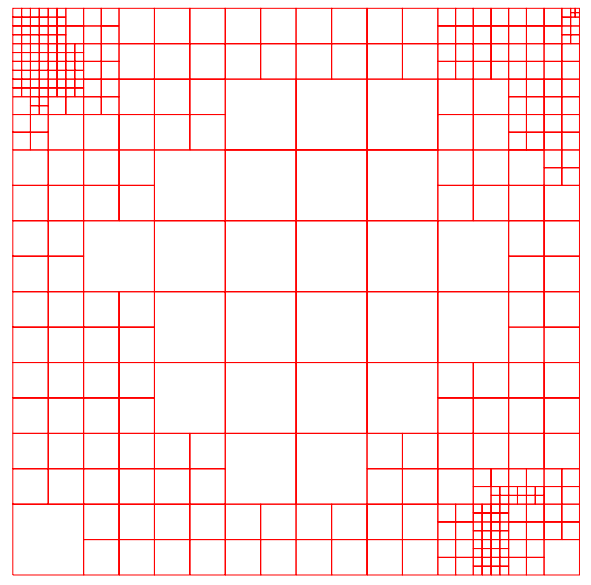

(a) mesh

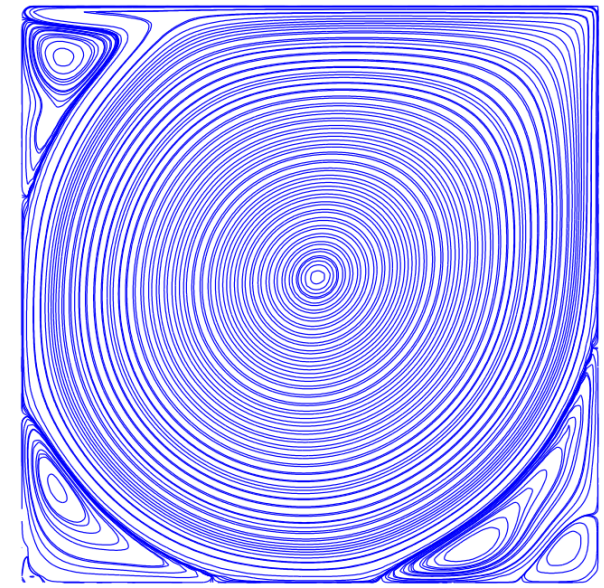

(b) streamlines

Figure 14: Streamlines for the cavity flow problem with $R e=10000$ on an adaptive quartic mesh after 6 refinements and using artificial time stepping with $\mathrm{dt}=1.0$; final mesh has 379 elements $(82,549 \mathrm{dofs})$. The energy error of the solution is $3.0 \times 10^{-4}$. Note that this is a steady state solution in a transient regime. 
reported by Botella and Peyret, and found that in every case our refined solutions agree well (in many cases, to three or four digits) with their reported numbers. In the interest of brevity, here we report a relevant subset of these results.

Perhaps the benchmark values of greatest engineering interest are the extremal velocities along the horizontal and vertical centerlines. Table 1 shows these values for a series of $h$-refinements on a quartic mesh, together with the benchmark values. As can be seen, even the coarsest mesh has values not too far from the benchmark, and as we refine the values approach those of the benchmark.

Also of interest are the center locations of the various vortices and the vorticity and the streamfunction values at those points. The vortex centers are local extrema of the streamfunction-the center of the primary vortex is a local maximum, the centers of secondary vortices are local minima, the centers of tertiary vortices are again local maxima, and so on. The values for the primary vortex center are shown in Table 2 . Here, only the vorticity value is off significantly in the coarsest $2 \times 2$ mesh, and after just one refinement, all values agree with the benchmark in the first digit. By the time we have completed 8 refinements, $\psi$ agrees to four digits, while $\omega$ agrees to three. It is worth noting, however, that in several cases, our solver differs in some of the apparently converged digits from those reported by Botella and Peyret-e.g., our $u_{\max }$ appears to have converged to four or five digits, and the fourth digit differs from that in [3]. This may simply reflect the difference in our respective boundary conditions-recall that we approximate the discontinuity at the top corners, while Botella and Peyret solve with discontinuous boundary conditions. Alternately, the discrepancy may reflect accuracy limitations of the solution in [3]; in any case, the difference warrants further investigation.

\begin{tabular}{cccllllll}
\hline Ref. Type & \# Refs & dofs & $u_{\max }$ & $y_{\max }$ & $v_{\max }$ & $x_{\max }$ & $v_{\min }$ & $x_{\min }$ \\
\hline Benchmark & - & - & 0.3885698 & 0.1717 & 0.3769447 & 0.8422 & -0.5270771 & 0.0908 \\
$h, k=4$ & 0 & 934 & 0.396339 & 0.242442 & 0.39888 & 0.812133 & -0.478214 & 0.131708 \\
$h, k=4$ & 1 & 3570 & 0.393731 & 0.197367 & 0.377642 & 0.825603 & -0.49186 & 0.10713 \\
$h, k=4$ & 2 & 6885 & 0.384899 & 0.179395 & 0.369079 & 0.832126 & -0.511888 & 0.0857587 \\
$h, k=4$ & 3 & 9561 & 0.385443 & 0.178651 & 0.371756 & 0.838819 & -0.523973 & 0.0934616 \\
$h, k=4$ & 4 & 13555 & 0.386732 & 0.177283 & 0.373124 & 0.839166 & -0.526561 & 0.0924524 \\
$h, k=4$ & 5 & 22182 & 0.387341 & 0.173529 & 0.37668 & 0.841985 & -0.528125 & 0.091373 \\
$h, k=4$ & 6 & 32027 & 0.388339 & 0.171578 & 0.376706 & 0.842174 & -0.527996 & 0.0905222 \\
$h, k=4$ & 7 & 47763 & 0.38832 & 0.171756 & 0.376777 & 0.842211 & -0.527 & 0.0907992 \\
$h, k=4$ & 8 & 62960 & 0.388328 & 0.17176 & 0.376816 & 0.842126 & -0.526955 & 0.0908152 \\
\hline
\end{tabular}

Table 1: Extrema of the velocity along vertical and horizontal centerlines for cavity flow with $R e=1000$. Benchmark values are the highest-fidelity results $(N=160$ case $)$ from Botella and Peyret [3].

\begin{tabular}{cccllll}
\hline Ref. Type & \# Refs & dofs & $\psi$ & $\omega$ & $x$ & $y$ \\
\hline Benchmark & - & - & 0.1189366 & 2.067753 & 0.4692 & 0.5652 \\
$h, k=4$ & 0 & 934 & 0.147864 & 6.41215 & 0.478689 & 0.595329 \\
$h, k=4$ & 1 & 3570 & 0.121863 & 2.2432 & 0.465454 & 0.568378 \\
$h, k=4$ & 2 & 6885 & 0.119 & 2.03875 & 0.468219 & 0.561995 \\
$h, k=4$ & 3 & 9561 & 0.119314 & 2.059 & 0.471577 & 0.564492 \\
$h, k=4$ & 4 & 13555 & 0.119715 & 2.06886 & 0.471538 & 0.563466 \\
$h, k=4$ & 5 & 22182 & 0.119161 & 2.06428 & 0.469261 & 0.564757 \\
$h, k=4$ & 6 & 32027 & 0.119052 & 2.06538 & 0.469334 & 0.564946 \\
$h, k=4$ & 7 & 47763 & 0.11896 & 2.06663 & 0.4692 & 0.5652 \\
$h, k=4$ & 8 & 62960 & 0.118957 & 2.0666 & 0.4692 & 0.5652 \\
\hline
\end{tabular}

Table 2: Location of the primary vortex center in the cavity flow problem, and values of streamfunction and vorticity at the vortex center for $\operatorname{Re}=1000$. Benchmark values are the highest-fidelity results $(N=160$ case $)$ from Botella and Peyret [3]. 


\subsection{Backward-Facing Step}

Another common model problem for Stokes and Navier-Stokes flow involves flow over a backward-facing step, a schematic of which can be seen in Figure 15. The corner induces a singularity in the stress, making this a challenging flow to resolve, and one well-suited to adaptivity. Following Gartling's benchmark paper [25], we consider the backward-facing step problem for $\mathrm{Re}=800$. At this Reynolds number, a steady state solution exists, but there are 3D effects, so 2D computations should be understood primarily as a mechanism for comparison rather than as predictive of real-world results. Gartling prescribes a parabolic velocity profile immediately above the step-i.e. in his computations the domain is rectangular, with wall conditions for the bottom half of the inflow end of the domain-this too is not reflective of conditions that could be replicated by an experiment, but useful for comparison. A schematic can be seen in Figure 16, and a streamline plot for the inflow region can be found in Figure 17.

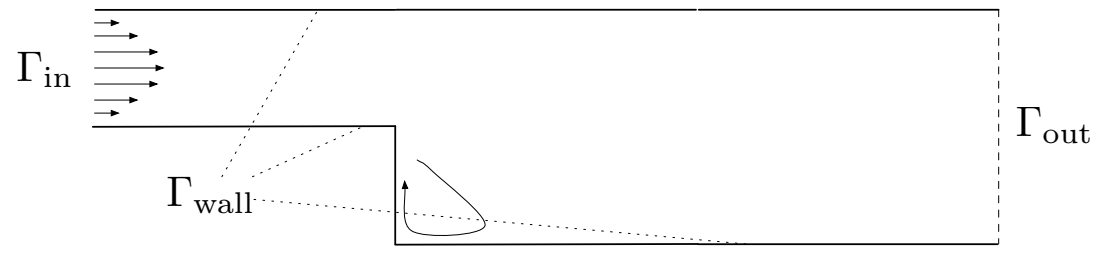

Figure 15: Schematic of the classic backward-facing step problem with a parabolic inflow profile.

With these parameters, there are two recirculation regions in the flow, one immediately east of the step, and one along the ceiling. The distance from the step to the reattachment point is known as the primary reattachment length; the distance from the inflow to the separation point on the ceiling is the secondary separation point, and the distance from the inflow to the ceiling reattachment point is the secondary reattachment point. These lengths are among the benchmark values reported by Gartling.

Our experiment proceeds as follows. We define a two-element preliminary mesh that covers the domain $(0,30) \times$ $(-0.5,0.5)$. We then refine the entire mesh anisotropically four times to produce a 32-element mesh with aspect ratio less than 4 in each element. This is the mesh on which we begin our computations. We start with a zero initial guess, and use an initial nonlinear stopping criterion of $\epsilon_{0}=10^{-4}$. Once we converge in the nonlinear iteration, we measure the error in each element, adapt accordingly, and update the nonlinear stopping criterion. The initial guess for the new mesh is the projection of the previous solution. We use $k=3$ as the polynomial order for field variables.

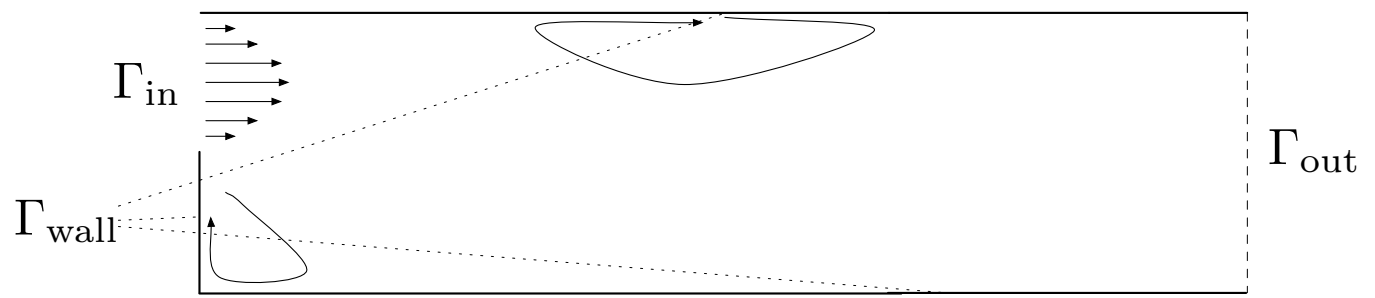

Figure 16: Schematic of the backward-facing step problem with the computational domain and inflow prescribed by Gartling [25]. Following Gartling, we take $\operatorname{Re}=800$ and the domain to be $(0,30) \times(-0.5,0.5)$. 


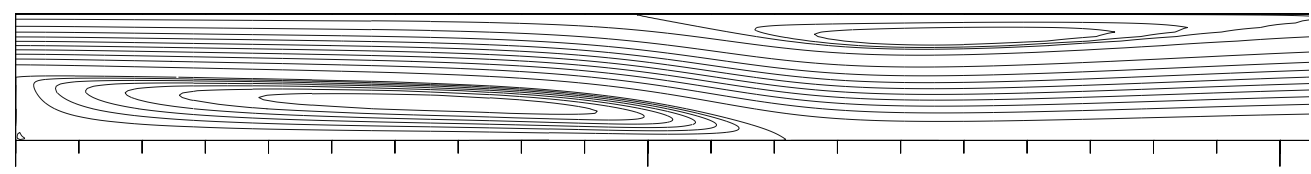

Figure 17: Streamlines for the backward-facing step problem for $\operatorname{Re}=800$ on the domain $(0,10.5) \times(-0.5,0.5)$.

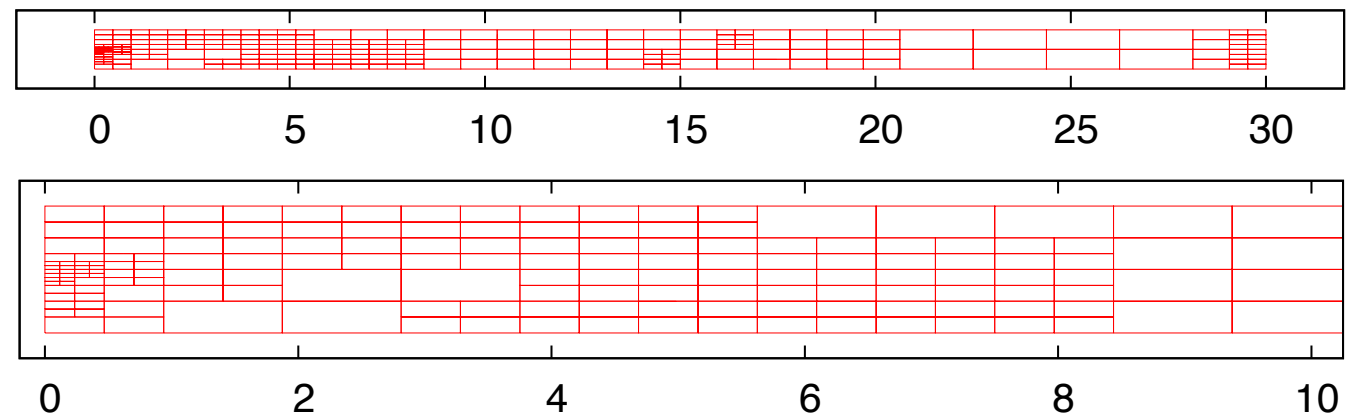

Figure 18: Mesh for the Gartling backward-facing step problem after 4 refinement steps, mesh and zoom near the inflow. The mesh has 167 elements and 24,568 degrees of freedom.

The separation and reattachment points are defined as points where the viscous shear stress is zero at the wall—that is, points where the wall-normal derivative of the wall-tangential velocity is zero. Thus, for our flow, we may measure the separation and reattachment lengths by searching along the ceiling and floor of the domain for points at which $\frac{\partial u_{1}}{\partial y}$ reverses sign. We perform such a search by using an initial point inside the recirculation region and one outside it. We compute a new point half the distance between these points, and compare the signs, and replace the original point with the one whose sign agrees. We proceed in this way for 30 iterations. Our initial points for the primary reattachment length are $(1,-0.5)$ and $(15,-0.5)$; for the secondary separation, $(1,0.5)$ and $(7,0.5)$; for the secondary reattachment, $(7,0.5)$ and $(15,0.5)$.

In Table 3, the results for the first 10 refinement steps are shown. On the initial mesh, we are unable to compute meaningful separation and reattachment lengths; in fact, the secondary separation computation fails because $\frac{\partial u_{1}}{\partial y}$ has the same sign at the initial points. Subsequent refinements, however, soon converge to values that agree with Gartling to the accuracy he provides. Moreover, the values appear to have converged to five or six digits in the final adaptive solution. Note, too, that although the nonlinear iteration takes as many as 27 steps to converge on the coarser meshes, only three or four iterations are required to converge to the increasingly fine tolerance required from the fourth refinement step onward. From the point of view of computational cost, this is an appealing state of affairs: the solves on the coarse meshes are quite cheap compared to those on the finest meshes. It is also worth noting that in this case, even the finest meshes are not particularly expensive: the finest mesh has 325,156 degrees of freedom, and of these, only 75,284 belong to the mesh skeleton-when using static condensation, the global solve thus involves only 75,284 degrees of freedom. Using four MPI processes on an early 2013 MacBook Pro laptop with a quad-core $2.7 \mathrm{GHz}$ Intel Core i7 (Sandy Bridge) processor, our driver completes the entire sequence of adaptive solves in about 13 minutes. 
Gartling also provides data for several quantities of interest along the lines $x=7$ and $x=15$. To the accuracy he provides, our adaptive solution agrees well after the fourth refinement or so. Figures 19 and 20 plot several of our adaptive solutions for $u_{1}, u_{2}$, and vorticity $\omega=\operatorname{Re}\left(\boldsymbol{\sigma}_{21}-\boldsymbol{\sigma}_{12}\right)$ alongside the data provided by Gartling. (We do not plot any adaptive solutions beyond the sixth refinement because these are visually indistinguishable from the sixth refinement.)
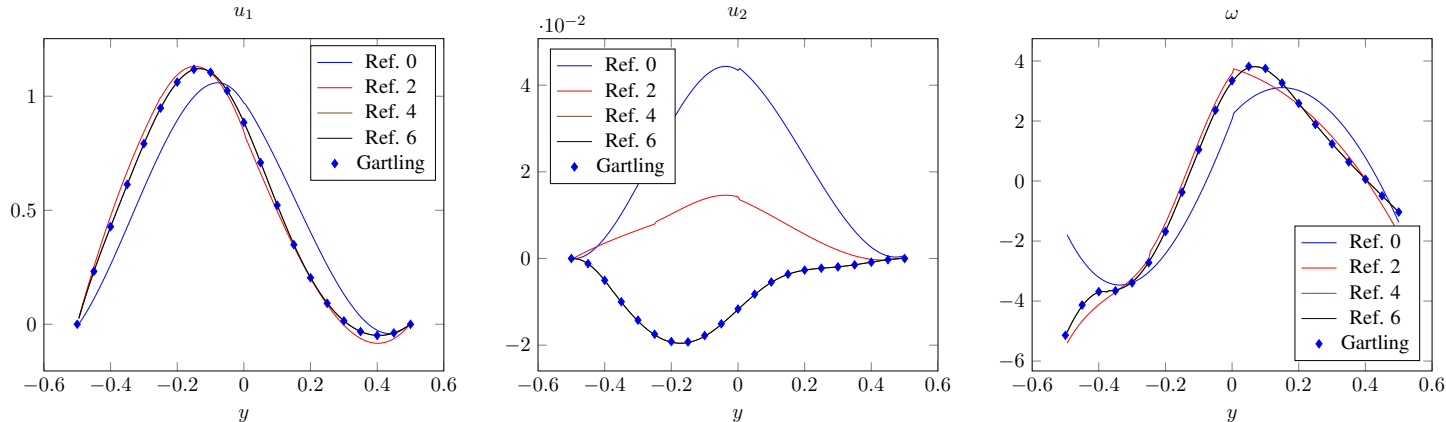

Figure 19: Backward-facing step: $u_{1}, u_{2}$, and $\omega$ values for $x=7$.
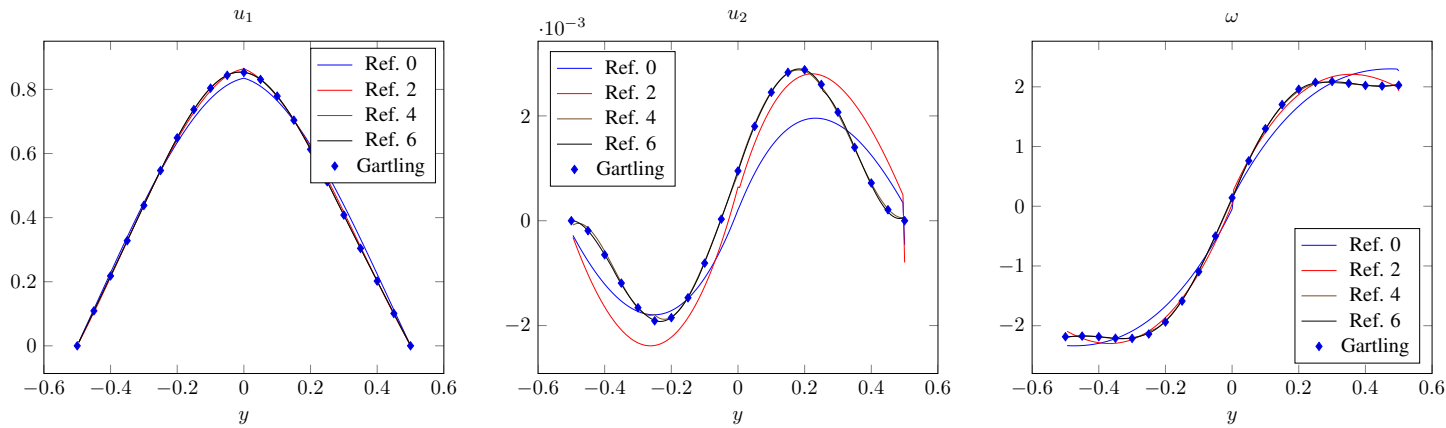

Figure 20: Backward-facing step: $u_{1}, u_{2}$, and $\omega$ values for $x=15$

\begin{tabular}{|c|c|c|c|c|c|c|c|c|}
\hline ref. \# (i) & $S_{1}$ & $S_{2}$ & $S_{3}$ & $e_{\mathrm{rel}}^{i}$ & $\epsilon_{i}$ & It. count & $N_{\mathrm{el}}$ & dofs \\
\hline Gartling & 6.10 & 4.85 & 10.48 & & & & & \\
\hline 0 & 1.72486599 & - & 8.38504412 & $1.56 \mathrm{E}-002$ & $1.00 \mathrm{E}-004$ & 22 & 32 & 4834 \\
\hline 1 & 3.87936803 & 2.14772006 & 9.25664368 & $2.41 \mathrm{E}-002$ & $1.56 \mathrm{E}-006$ & 27 & 44 & 6602 \\
\hline 2 & 5.69180839 & 4.32280832 & 10.40353156 & $1.38 \mathrm{E}-002$ & $2.41 \mathrm{E}-006$ & 21 & 80 & 11906 \\
\hline 3 & 6.10015343 & 4.86189019 & 10.46046167 & $6.69 \mathrm{E}-003$ & $1.38 \mathrm{E}-006$ & 7 & 167 & 24548 \\
\hline 4 & 6.09635072 & 4.85762446 & 10.46496340 & $3.88 \mathrm{E}-003$ & $6.69 \mathrm{E}-007$ & 3 & 248 & 36370 \\
\hline 5 & 6.09710370 & 4.85788347 & 10.46850055 & $2.21 \mathrm{E}-003$ & $3.88 \mathrm{E}-007$ & 4 & 350 & 51350 \\
\hline 6 & 6.09638997 & 4.85738019 & 10.47732290 & $1.34 \mathrm{E}-003$ & $2.21 \mathrm{E}-007$ & 4 & 524 & 76762 \\
\hline 7 & 6.09638496 & 4.85334930 & 10.47719502 & $8.13 \mathrm{E}-004$ & $1.34 \mathrm{E}-007$ & 3 & 812 & 118682 \\
\hline 8 & 6.09608075 & 4.85295549 & 10.47823676 & $4.21 \mathrm{E}-004$ & $8.13 \mathrm{E}-008$ & 3 & 1190 & 173414 \\
\hline 9 & 6.09600439 & 4.85293330 & 10.47919434 & $2.04 \mathrm{E}-004$ & $4.21 \mathrm{E}-008$ & 3 & 1817 & 265152 \\
\hline 10 & 6.09601543 & 4.85295562 & 10.47917082 & $1.41 \mathrm{E}-004$ & $2.04 \mathrm{E}-008$ & 3 & 2231 & 325156 \\
\hline
\end{tabular}

Table 3: Reattachment and separation lengths for Re $=800$ backward-facing step problem with $k=3$, refinements 0 - 10 . $S_{1}$ : primary reattachment; $S_{2}$ : secondary separation; $S_{3}$ : secondary reattachment. $e_{\text {rel }}^{i}$ is the relative energy error for the $i$ th adaptive solution, which is used to determine $\epsilon_{i+1}$, the nonlinear stopping tolerance for the next refinement. 
In the hope of offering a higher-precision benchmark, we repeat the experiment, now for 13 refinement steps. The final mesh has 4040 elements, with 587,650 degrees of freedom, of which 135,170 belong to the mesh skeleton. We provide our detailed results for $x=7$ and $x=15$ in Appendix Appendix A.

\subsection{Flow Past a Cylinder}

Another common model problem for the Navier-Stokes equations is flow past a cylinder. For Reynolds numbers between 6 and 40, a steady state solution exists with standing vortices in the cylinder wake. At some critical Reynolds number $\operatorname{Re}_{c}$ above 40, the flow becomes unsteady. Kovasznay performed some early experiments, suggesting $\operatorname{Re}_{c}=$ 40 [33]; recently, Kalita and Sen have argued, by a combination of numerical simulations and lab experiments, that $46.5<\operatorname{Re}_{c} \leq 47$; they estimate $\operatorname{Re}_{c} \approx 46.5$ [30].

The geometry of the problem is as shown in Figure 21; we take the domain to be a large enough box relative to the cylinder that free stream conditions may be assumed at the inflow and top and bottom of the domain. The Reynolds number is defined as

$$
\operatorname{Re}=\frac{U_{\infty} D}{\nu}
$$

for a free stream velocity $U_{\infty}$, cylinder diameter $D$, and viscosity $\nu$.

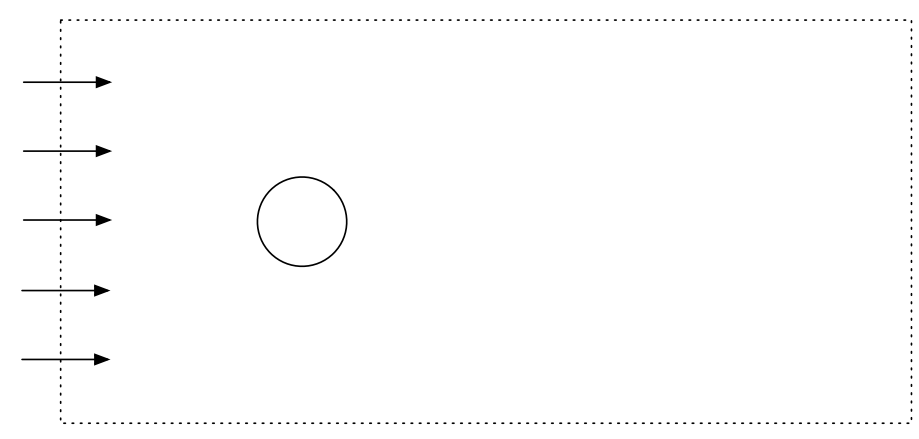

Figure 21: Schematic of the flow past a cylinder problem.

Following Kalita and Sen, we take the free stream velocity to be constant: $U_{\infty}=1$. We accordingly impose Dirichlet boundary conditions on the velocity trace $\widehat{\boldsymbol{u}}=\left(\begin{array}{c}U_{\infty} \\ 0\end{array}\right)$ at the inflow and top and bottom of the domain; we impose zero-traction (“do-nothing") conditions $\widehat{\boldsymbol{t}}_{n}=0$ on the outflow.

Our main adaptive experiments are as follows. We take the cylinder to have unit diameter. On a 240 by 120 domain, we use Dirichlet conditions on the inflow velocity and using penalty constraints we impose zero tractions on the top, bottom, and outflow boundaries. We begin with a cubic mesh that simply captures the geometry, shown in Figure 22(a), then perform some initial refinements to ensure that element aspect ratios are less than 2 and to produce a 1-irregular mesh, shown in Figure 22(b). We use our adaptive nonlinear stopping criterion with $\epsilon_{0}=10^{-5}$. After 4 refinements, our mesh has 2712 elements, with 392,592 degrees of freedom. The final mesh is shown in Figure 23. 
The results are shown in Table 4. We then repeat this experiment on a 480 by 240 domain; the results are shown in Table 5. From the agreement between finer and coarser meshes and between the larger and smaller domains, it appears we can claim about three digits of accuracy in each of the quantities of interest.

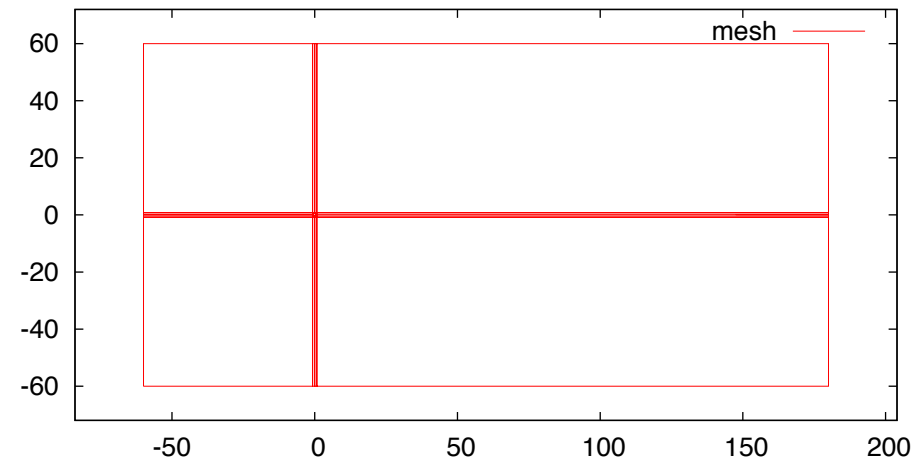

(a) preliminary mesh

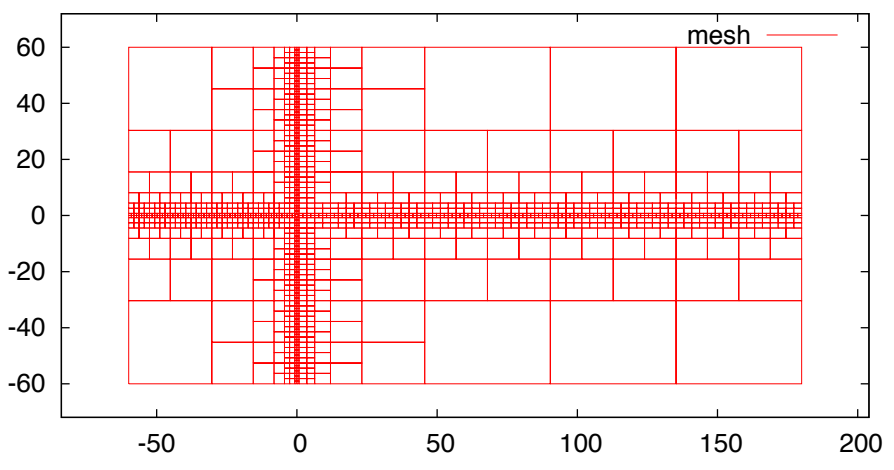

(b) initial mesh

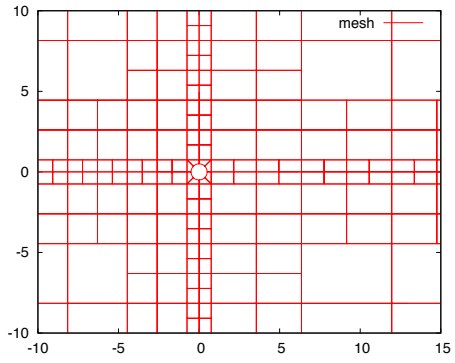

(c) initial mesh

Figure 22: Preliminary cubic mesh for the flow past a cylinder problem at $\mathrm{Re}=40$, and the mesh after some initial refinements were done to ensure that the mesh is roughly isotropic.

Some quantities of interest for this problem are as follows:

- angle of separation $\theta_{s}$

- wake length $l$

- drag coefficient $C_{D}$

We can define drag and lift forces

$$
F_{D}=\int_{S} f_{\text {friction }} n_{x}-p n_{x}, \quad F_{L}=\int_{S}-\left(f_{\text {friction }} n_{x}+p n_{y}\right)
$$

where the integral is around the circle $S, \boldsymbol{n}=\left(\begin{array}{l}n_{x} \\ n_{y}\end{array}\right)$ is the outward normal, and $f_{\text {friction }} \stackrel{\text { def }}{=}(\boldsymbol{\sigma n}) \times \boldsymbol{n}$. The drag and 


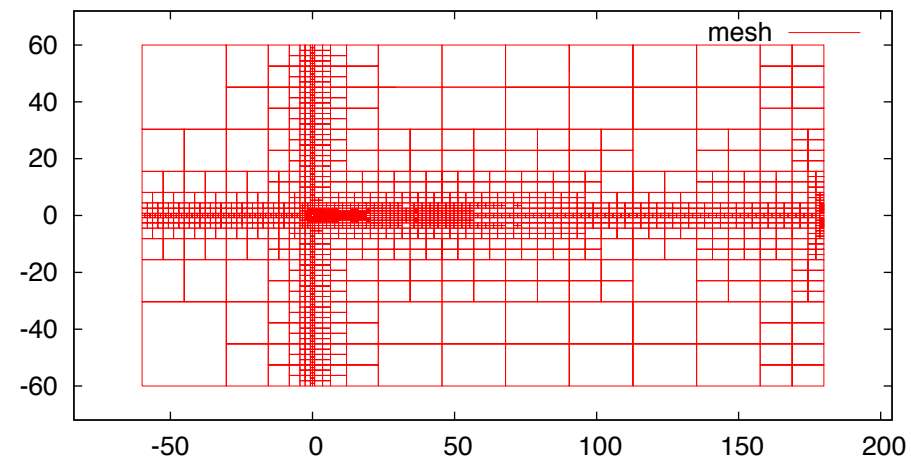

(a) final mesh

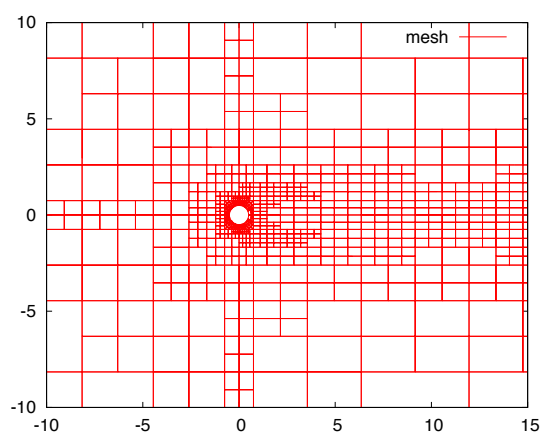

(b) final mesh, detail

Figure 23: Cubic mesh for the flow past a cylinder problem at $R e=40$ : mesh after 4 refinements, and a detail of that mesh. 


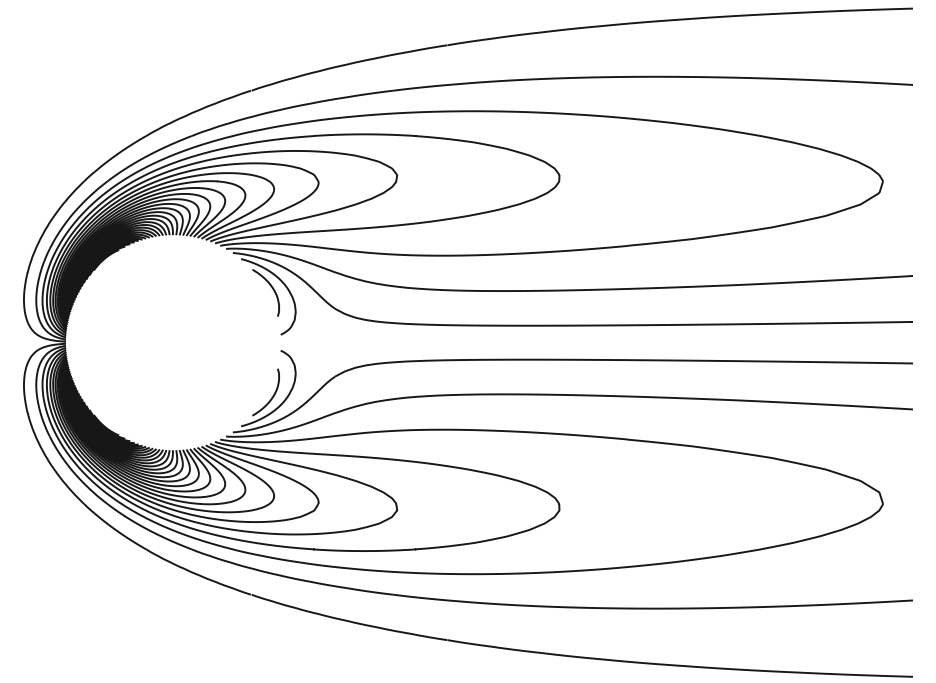

(a) vorticity

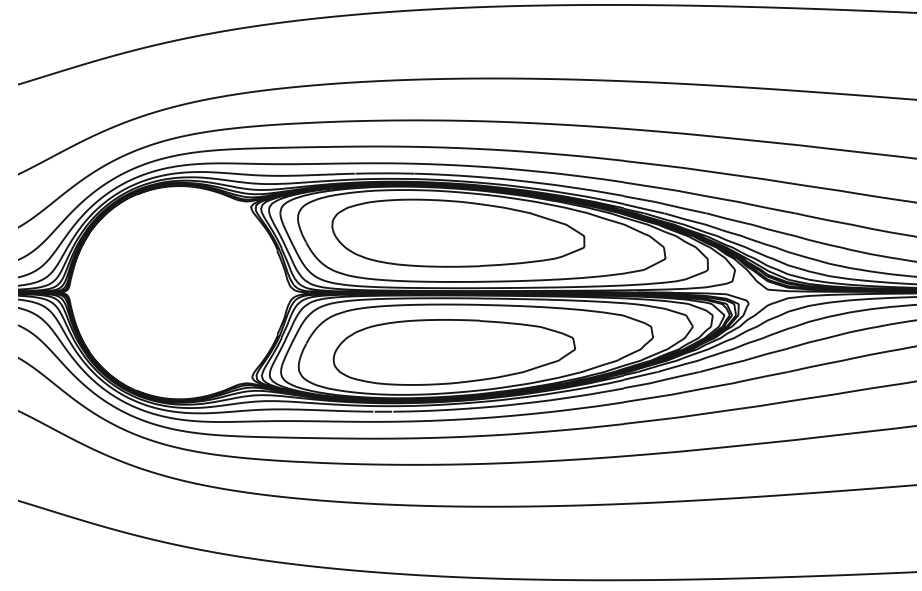

(b) stream function

Figure 24: Vorticity and stream function contours for the cylinder flow problem with $k=3$ after 4 refinements, detail near the cylinder 
lift coefficients are then defined as

$$
C_{D}=\frac{F_{D}}{\frac{1}{2} \rho U_{\infty}^{2} D}, \quad C_{L}=\frac{F_{L}}{\frac{1}{2} \rho U_{\infty}^{2} D} .
$$

Thus, for our computation, in which we have non-dimensionalized in a manner that gives us unit-valued $U_{\infty}, D$, and $\rho$, the values are simply $C_{D}=2 F_{D}$ and $C_{L}=2 F_{L}$.

We can determine the separation angle and wake length using a similar approach to the one we adopted to determine separation and reattachment points for the backward-facing step. For the separation angle, we search the arc on the cylinder between $\theta=\frac{\pi}{8}$ and $\theta=\frac{3 \pi}{8}$ for a point at which the viscous shear stress is zero; for the wake length, we search along the line from $(2,0)$ to $(180,0)$ for a point at which $u_{1}=0$. We compute these values for each adaptive solution. The results are shown in Table 4.

\begin{tabular}{cccccccccr}
\hline ref. \# $(i)$ & $l$ & $\theta_{s}$ & $C_{D}$ & $e_{\text {rel }}^{i}$ & $\epsilon_{i}$ & It. count & $N_{\text {el }}$ & dofs & trace dofs \\
\hline 0 & 4.12911 & $49.4341^{\circ}$ & 1.38701 & $4.41 \mathrm{E}-002$ & $1.00 \mathrm{E}-005$ & 7 & 1524 & 221656 & 50968 \\
1 & 4.44991 & $51.7753^{\circ}$ & 1.50047 & $1.13 \mathrm{E}-002$ & $4.41 \mathrm{E}-007$ & 4 & 1584 & 230400 & 52992 \\
2 & 4.46920 & $51.8212^{\circ}$ & 1.50497 & $3.88 \mathrm{E}-003$ & $1.13 \mathrm{E}-007$ & 3 & 1668 & 242552 & 55736 \\
3 & 4.49579 & $53.2717^{\circ}$ & 1.50550 & $1.42 \mathrm{E}-003$ & $3.88 \mathrm{E}-008$ & 3 & 1974 & 286756 & 65668 \\
4 & 4.49609 & $53.2714^{\circ}$ & 1.50541 & $5.70 \mathrm{E}-004$ & $1.42 \mathrm{E}-008$ & 3 & 2712 & 392592 & 88848 \\
5 & 4.49610 & $53.2715^{\circ}$ & 1.50541 & $3.89 \mathrm{E}-004$ & $5.70 \mathrm{E}-009$ & 3 & 3090 & 447324 & 101244 \\
6 & 4.49796 & $53.2715^{\circ}$ & 1.50538 & $2.45 \mathrm{E}-004$ & $3.89 \mathrm{E}-009$ & 3 & 3702 & 535604 & 120980 \\
7 & 4.49797 & $53.5405^{\circ}$ & 1.50532 & $1.50 \mathrm{E}-004$ & $2.45 \mathrm{E}-009$ & 3 & 4470 & 646548 & 145908 \\
\hline
\end{tabular}

Table 4: Flow past a cylinder problem: quantities of interest for four adaptive refinement steps, using a $240 \times 120$ domain with cubic field variables.

\begin{tabular}{ccccccccrr}
\hline ref. \# $(i)$ & $l$ & $\theta_{s}$ & $C_{D}$ & $e_{\text {rel }}^{i}$ & $\epsilon_{i}$ & It. count & $N_{\text {el }}$ & total dofs & trace dofs \\
\hline 0 & 4.00566 & $49.2610^{\circ}$ & 1.34084 & $4.53 \mathrm{E}-002$ & $1.00 \mathrm{E}-005$ & 7 & 3112 & 452272 & 103728 \\
1 & 4.42990 & $51.7317^{\circ}$ & 1.49247 & $1.13 \mathrm{E}-002$ & $4.53 \mathrm{E}-007$ & 4 & 3172 & 461016 & 105752 \\
2 & 4.45697 & $51.7952^{\circ}$ & 1.49997 & $3.85 \mathrm{E}-003$ & $1.13 \mathrm{E}-007$ & 3 & 3256 & 473168 & 108496 \\
3 & 4.48506 & $53.2428^{\circ}$ & 1.50106 & $1.40 \mathrm{E}-003$ & $3.85 \mathrm{E}-008$ & 3 & 3562 & 517340 & 118396 \\
4 & 4.48549 & $53.2429^{\circ}$ & 1.50102 & $5.81 \mathrm{E}-004$ & $1.40 \mathrm{E}-008$ & 3 & 4426 & 641100 & 145388 \\
5 & 4.48737 & $53.2430^{\circ}$ & 1.50099 & $3.13 \mathrm{E}-004$ & $5.81 \mathrm{E}-009$ & 3 & 5446 & 787844 & 177892 \\
6 & 4.48738 & $53.5141^{\circ}$ & 1.50094 & $1.95 \mathrm{E}-004$ & $3.13 \mathrm{E}-008$ & 3 & 6190 & 895140 & 201860 \\
7 & 4.48739 & $53.5141^{\circ}$ & 1.50093 & $1.32 \mathrm{E}-004$ & $1.95 \mathrm{E}-009$ & 2 & 6994 & 1010860 & 227532 \\
\hline
\end{tabular}

Table 5: Flow past a cylinder problem: quantities of interest for seven adaptive refinement steps, using a $480 \times 240$ domain with cubic field variables.

Experimental evidence for the value of $C_{D}$ at $\mathrm{Re}=40$. The values reported in the literature of the quantities of interest for this problem are surprisingly varied; several of these are listed in Table 6 . The situation with regard to the drag coefficient $C_{D}$ is the most striking, because several papers which differ significantly in $C_{D}$ claim agreement with the same experiment. In 1959, Tritton reported the results of an experimental study to ascertain the relationship between Reynolds number and drag coefficient $C_{D}$ for the flow past a cylinder problem [53]. Prabhakar and Reddy [45], Pontaza and Reddy [44], and Le, Khoo, and Peraire [34] each cite Tritton. Pontaza and Reddy report $C_{D}$ of 1.5537 while Prabhakar and Reddy report $C_{D}$ of 1.55 ; both papers claim good agreement with Tritton, who they claim reported a value of 1.56. Strikingly, Le et al. report Tritton's drag coefficient for the same Reynolds number as 1.48. Each of these claims elides Tritton's results, in effect neglecting his error bars. Tritton plots an estimated mean curve; the experimental points nearest Reynolds 40 are $\operatorname{Re}=38.5, C_{D}=1.46$ and $\operatorname{Re}=41.7, C_{D}=1.62$. Tritton further suggests his results have an expected accuracy of 6\%. Examining the plot in [53, Fig. 1], the value of $C_{D}$ corresponding to $\operatorname{Re}=40$ is about 1.58 . Taking a $6 \%$ interval around this value would yield a range of 1.49 to 1.68 . 
Reported values in the numerical literature vary from 1.498 to $1.62-$ we thus may take all these values to agree with Tritton.

\begin{tabular}{llll}
\hline & $l$ & $\theta_{s}$ & $C_{D}$ \\
\hline Takami \& Keller (1969) [52] & 4.65 & $53.55^{\circ}$ & 1.5359 \\
Dennis \& Chang (1970) [23] & 4.69 & $53.8^{\circ}$ & 1.522 \\
Nieuwstadt \& Keller (1973) [40] & 4.357 & & 1.550 \\
Loc (1975) [35] & 4.27 & & 1.494 \\
Coutanceau \& Bouard (1977, experiment) [15] & 3.78 & $52.8^{\circ}$ & - \\
Fornberg (1980) [24] & 4.48 & $\approx 54^{\circ}$ & 1.4980 \\
Loc (1980) [36] & 4.27 & - & \\
He \& Doolen (1997) [28] & 4.49 & $52.84^{\circ}$ & 1.499 \\
Ye et al. (1999) [55] & 4.54 & - & 1.52 \\
Calhoun (2002) [11] & 4.36 & $54.2^{\circ}$ & 1.62 \\
Niu et al. (2003) [41] & 4.51 & $52.84^{\circ}$ & 1.574 \\
Pontaza \& Reddy (2003) [44] & 4.55 & - & 1.5537 \\
Russell \& Wang (2003) [50] & 4.58 & $53.1^{\circ}$ & 1.60 \\
Sanyasiraju \& Manjula (2005) [51] & 4.21 & $51.0249^{\circ}$ & 1.5308 \\
Le et al. (2006) [34] & 4.44 & $53.6^{\circ}$ & 1.56 \\
Prabhakar \& Reddy (2006) [45] & 4.55 & - & 1.55 \\
Kalita \& Sen (2012) [30] & 4.433 & $53.270^{\circ}$ & 1.590 \\
Present & 4.49 & $53.2^{\circ}$ & 1.50 \\
\hline
\end{tabular}

Table 6: Quantities of interest for the flow past a cylinder problem reported in the literature for $\operatorname{Re}=40$.

\section{Conclusions and Future Work}

In this paper, we have applied DPG to several model problems in steady 2D incompressible flow. Along the way, we developed a DPG formulation linearized Navier-Stokes equations, and verified the approach using the Kovasznay manufactured solution, achieving optimal convergence rates.

We demonstrated the effectiveness of DPG's energy error measurement for robust adaptivity in the context of a variety of flow problems: the lid-driven cavity flow problem, the backward-facing step problem, and the flow past a cylinder problem.

Underpinning all of these efforts was Camellia, the software framework for rapid development of DPG solvers which we developed for our research—offering convenient, modular interfaces for the definition of DPG bilinear forms and test space norms, providing simple, extensible mechanisms for $h$-, $p$-, and $h p$-adaptivity, and taking advantage of the embarrassingly parallel nature of optimal test function determination to provide scalable computation of the stiffness matrix, among other features.

These results demonstrate DPG's great promise in the context of incompressible flow problems: the fact that the method is stable even on coarse meshes combined with the built-in error measure means that we may begin with a mesh that merely captures the geometry and perform automatic refinements to converge to a solution with desired accuracy. While the present numerical experiments are limited to two-dimensional, steady-state problems, both our analysis and the numerical results thus far warrant optimism for DPG applied to three-dimensional and transient problems. If fully realized, the capabilities of DPG we have explored here could eliminate the need to design complex grids to resolve expected features of the solution. 


\section{Future Research Directions}

Our research efforts thus far suggest several fruitful areas for future exploration.

\section{1. $D P G$ and $H P C$}

DPG has several features that make it a good candidate for future high-performance computing (HPC) applications. First, it is a high-order method; high-order methods are known to provide better computational intensity-a measure of the amount of computational effort expended relative to the amount of communication required. Since the progression in HPC is toward higher relative communications costs- "flops will be free" has become a tagline in discussions of exascale computing - higher computational intensity is a desirable feature. DPG's optimal test function computation, being an element-local operation, similarly adds to the computational intensity-DPG's optimal test functions allow one to trade local effort for improved accuracy. Camellia's use of static condensation to reduce the global system to traces and flux coefficients also adds to the computational intensity: no information about field coefficients needs to be communicated between computational nodes.

The robust adaptivity offered by DPG also lends itself to HPC applications, in that it affords a level of automaticity uncommon in fluid dynamics computations_-and, as discussed in the introduction, in typical applications, the NavierStokes solve is just one component in an optimization loop, which makes failures that require human intervention costly. HPC increases the speed at which computations can in principle be completed, magnifying the cost of any events that halt the computation.

One of the key areas that must be addressed for DPG to be applied to many HPC-scale problems is the development of a DPG solver that scales to thousands—if not tens of thousands, or hundreds of thousands—of processors. As mentioned above, Camellia already provides a highly scalable mechanism for computing the optimal test functions and the global stiffness matrix; however, how to solve the global matrix system in a robust, efficient way remains an open question for most problems - a notable exception is the Poisson problem, for which Barker et al. have demonstrated that additive Schwarz preconditioners are effective [1].

There are a few clear lines of research which one might pursue in this context. First, one might build on recent work by Wieners and Wohlmuth [54] to develop a general framework for preconditioning the DPG stiffness matrix. Another

recent, and very promising, idea has recently been proposed by Bui-Thanh and Ghattas [10], who have developed a PDE-constrained optimization approach to DPG, providing a unified method for linear and nonlinear problems-and an iterative solver for DPG. Finally, one might take advantage of the rich geometric information that our approach has inherent within it—-together with the robustness of the method on coarse meshes—by adopting a geometric multigrid preconditioner for a conjugate gradient iteration. This last is a present area of focus for us; we hope to report initial results in the near future.

\subsection{Extending Camellia to More Dimensions: $3 D$ and Time}

Several of our studies in this paper have been regime-limited in the sense that at a critical Reynolds number a flow becomes three-dimensional or transient. Furthermore, certain fluid phenomena—notably vortex stretching-only 
arise in three-dimensional flows. Therefore, we would like to add support for 3D elements of various topological types - tetrahedra, hexahedra, and pyramids, perhaps-to Camellia.

We would also like to provide some mechanism for transient solves. We plan to add support for space-time elements,${ }^{8}$ which compute on a time slab-a tensor product structure, extruding the spatial mesh in the time dimension. The time slab may be refined in both time and space dimensions. Solution coefficients are only stored for the current time slab-a relatively modest additional cost, given that by this mechanism one can bring the entire DPG apparatus to bear on the time dimension. Our immediate plan in this regard is to add support for space-time elements to Camellia, by adding a mechanism for temporal extrusion of arbitrary element discretizations - thereby allowing transient computations for all element types supported by Camellia. ${ }^{9}$

\subsection{Variations on the DPG Theme}

We would like to add support for continuous field variables and a Python interface to Camellia, to enhance the breadth of Camellia's applicability and the ease with which end users may take advantage of it. This will allow both easier comparison with existing finite element technologies, as well as exploration of new variations on the DPG theme- the usual DPG methodology requires a discontinuous test space, for example, but there is no essential requirement that the trial space be discontinuous. It is even possible to pursue a DPG-like scheme that involves continuous test functions_-such an approach has recently been investigated by Dahmen et al. [16], as well as Broersen and Stevenson [7]. Having continuous finite elements available within Camellia would also facilitate certain forms of post-processing, notably the streamfunction solves in this paper (which we simply solved using DPG, which was not the most appropriate choice).

\subsection{Conclusion}

In this paper, we have demonstrated the applicability of DPG to several problems in incompressible fluid flow. We have thereby begun to deliver on DPG's great promise of accurate solutions, automatic stability, and robust adaptivity. We look forward to continuing this work, applying DPG and related methodologies to larger-scale problems and problems in three-dimensional and transient regimes.

License. The submitted manuscript has been created by UChicago Argonne, LLC, Operator of Argonne National Laboratory (“Argonne”). Argonne, a U.S. Department of Energy Office of Science laboratory, is operated under Contract No. DE-AC02-06CH11357. The U.S. Government retains for itself, and others acting on its behalf, a paidup nonexclusive, irrevocable worldwide license in said article to reproduce, prepare derivative works, distribute copies to the public, and perform publicly and display publicly, by or on behalf of the Government.

Acknowledgments. The first author is supported in this work by the U.S. Department of Energy, Basic Energy Sciences, Office of Science, under Contract No. DE-AC02-06CH11357. All three authors were also supported by the Department of Energy [National Nuclear Security Administration] under Award No. DE-FC52-08NA28615.

\footnotetext{
${ }^{8}$ In 2011, Chan et al. solved a transient 1D convection-dominated diffusion problem using DPG with space-time elements [14].

${ }^{9}$ It is worth noting that standard time-stepping approaches, such as a Crank-Nicolson method, are also possible in the context of DPG. Our preference is to take advantage of DPG's rich analytic apparatus to provide automatic adaptivity in the time dimension as well, but our initial experiments with standard techniques also appear promising.
} 
Provenance. Portions of this work are closely derived from the first author's dissertation [46]. In particular, Section 2 is derived from Chapter 2 of the dissertation, while Sections 3 and 4 are outgrowths of the work presented in Chapters 8 and 10 , respectively.

\section{Appendix A. Benchmark Results for Re=800 Backward-Facing Step Problem}

In the hope of offering a higher-precision benchmark than has previously been available in the literature, in Tables A.7-A.10 we provide detailed results from the 13-refinement experiment mentioned at the end of Section 3.3. The pressure values provided are relative to the upper left corner of the domain - that is, we take $p=0$ at $(0,0.5) .{ }^{10}$ The derivative data we provide is computed in terms of our variable $\sigma=\frac{1}{\operatorname{Re}} \nabla \boldsymbol{u}$. To give a rough sense for how well a given value has converged, we also provide the difference between the thirteenth and the twelfth refinement steps. The final mesh has 4040 elements, with 587,650 degrees of freedom, of which 135,170 belong to the mesh skeleton.

\begin{tabular}{rrrrrrrrr}
\hline$y$ & $u_{1}$ & diff. & $u_{2}$ & diff. & $p$ & diff. & $\omega$ & diff. \\
\hline 0.50 & $-9 \mathrm{e}-7$ & $9 \mathrm{e}-8$ & $1.45034 \mathrm{e}-8$ & $-3 \mathrm{e}-9$ & 0.117353 & $1 \mathrm{e}-6$ & -1.032455 & $2 \mathrm{e}-5$ \\
0.45 & -0.038140 & $2 \mathrm{e}-7$ & $-2.69060 \mathrm{e}-4$ & $-1 \mathrm{e}-7$ & 0.117365 & $1 \mathrm{e}-6$ & -0.491516 & $-3 \mathrm{e}-6$ \\
0.40 & -0.048920 & $-2 \mathrm{e}-7$ & $-8.56902 \mathrm{e}-4$ & $-1 \mathrm{e}-7$ & 0.117368 & $1 \mathrm{e}-6$ & 0.063577 & $-1 \mathrm{e}-5$ \\
0.35 & -0.031530 & $4 \mathrm{e}-8$ & $-1.47056 \mathrm{e}-3$ & $2 \mathrm{e}-9$ & 0.117363 & $1 \mathrm{e}-6$ & 0.638052 & $-1 \mathrm{e}-6$ \\
0.30 & 0.015146 & $2 \mathrm{e}-8$ & $-1.93266 \mathrm{e}-3$ & $-9 \mathrm{e}-9$ & 0.117356 & $1 \mathrm{e}-6$ & 1.242062 & $-2 \mathrm{e}-7$ \\
0.25 & 0.092920 & $3 \mathrm{e}-8$ & $-2.26109 \mathrm{e}-3$ & $-2 \mathrm{e}-8$ & 0.117375 & $1 \mathrm{e}-6$ & 1.892744 & $4 \mathrm{e}-7$ \\
0.20 & 0.204102 & $8 \mathrm{e}-8$ & $-2.70439 \mathrm{e}-3$ & $-4 \mathrm{e}-8$ & 0.117489 & $1 \mathrm{e}-6$ & 2.586753 & $5 \mathrm{e}-7$ \\
0.15 & 0.349293 & $-4 \mathrm{e}-6$ & $-3.66623 \mathrm{e}-3$ & $9 \mathrm{e}-8$ & 0.117834 & $1 \mathrm{e}-6$ & 3.251362 & $-3 \mathrm{e}-5$ \\
0.10 & 0.522995 & $1 \mathrm{e}-7$ & $-5.51380 \mathrm{e}-3$ & $-7 \mathrm{e}-8$ & 0.118641 & $1 \mathrm{e}-6$ & 3.717214 & $3 \mathrm{e}-6$ \\
0.05 & 0.709881 & $3 \mathrm{e}-7$ & $-8.32496 \mathrm{e}-3$ & $-2 \mathrm{e}-7$ & 0.120206 & $1 \mathrm{e}-6$ & 3.776135 & $-1 \mathrm{e}-5$ \\
0.00 & 0.885869 & $7 \mathrm{e}-7$ & $-1.17653 \mathrm{e}-2$ & $-2 \mathrm{e}-7$ & 0.122785 & $1 \mathrm{e}-6$ & 3.301869 & $3 \mathrm{e}-5$ \\
-0.05 & 1.024660 & $-5 \mathrm{e}-6$ & $-1.51925 \mathrm{e}-2$ & $2 \mathrm{e}-8$ & 0.126436 & $1 \mathrm{e}-6$ & 2.331957 & $2 \mathrm{e}-4$ \\
-0.10 & 1.105488 & $6 \mathrm{e}-6$ & $-1.79126 \mathrm{e}-2$ & $-4 \mathrm{e}-7$ & 0.130925 & $1 \mathrm{e}-6$ & 1.034655 & $8 \mathrm{e}-5$ \\
-0.15 & 1.117745 & $4 \mathrm{e}-7$ & $-1.93802 \mathrm{e}-2$ & $-3 \mathrm{e}-7$ & 0.135759 & $1 \mathrm{e}-6$ & -0.368046 & $-6 \mathrm{e}-5$ \\
-0.20 & 1.061892 & $-8 \mathrm{e}-9$ & $-1.92913 \mathrm{e}-2$ & $-2 \mathrm{e}-7$ & 0.140346 & $1 \mathrm{e}-6$ & -1.664264 & $1 \mathrm{e}-6$ \\
-0.25 & 0.947822 & $-3 \mathrm{e}-7$ & $-1.75902 \mathrm{e}-2$ & $-2 \mathrm{e}-7$ & 0.144183 & $1 \mathrm{e}-6$ & -2.691594 & $-2 \mathrm{e}-6$ \\
-0.30 & 0.791738 & $-6 \mathrm{e}-7$ & $-1.44277 \mathrm{e}-2$ & $-6 \mathrm{e}-8$ & 0.146996 & $1 \mathrm{e}-6$ & -3.360474 & $7 \mathrm{e}-5$ \\
-0.35 & 0.612679 & $-9 \mathrm{e}-6$ & $-1.00613 \mathrm{e}-2$ & $-8 \mathrm{e}-7$ & 0.148764 & $1 \mathrm{e}-6$ & -3.641761 & $-3 \mathrm{e}-4$ \\
-0.40 & 0.427237 & $-7 \mathrm{e}-7$ & $-5.08039 \mathrm{e}-3$ & $-9 \mathrm{e}-8$ & 0.149672 & $1 \mathrm{e}-6$ & -3.717351 & $8 \mathrm{e}-6$ \\
-0.45 & 0.231945 & $-4 \mathrm{e}-7$ & $-1.19252 \mathrm{e}-3$ & $-2 \mathrm{e}-8$ & 0.150017 & $1 \mathrm{e}-6$ & -4.169665 & $8 \mathrm{e}-6$ \\
-0.50 & $-3 \mathrm{e}-6$ & $-2 \mathrm{e}-10$ & $-2.23131 \mathrm{e}-7$ & $-2 \mathrm{e}-10$ & 0.150108 & $1 \mathrm{e}-6$ & -5.152468 & $9 \mathrm{e}-6$ \\
\hline
\end{tabular}

Table A.7: Back-facing step after 13 refinements, data for $\mathrm{x}=7$. The values in the "diff." columns are the differences between the value after 13 refinements and the value after 12 refinements.

\footnotetext{
${ }^{10}$ Gartling chooses instead to scale his pressure relative to the origin, which is the bottom of the inflow, precisely at the geometric singularity. Because this is perhaps the single point at which computational methods are least likely to obtain a precise value for the pressure, the choice seems inauspicious. When we likewise scale our pressure relative to the origin, we find we differ significantly from Gartling's values; for instance, at $(15,0)$ our pressure is 0.2957 , compared with Gartling's 0.2459 . However, if we look at the difference between the pressure at $(15,0)$ and that at $(15,0.5)$, say, we find that we agree with Gartling — the difference is about 0.0001 . The obvious explanation is that our pressure solution agrees with Gartling essentially everywhere but at the origin.
} 


\begin{tabular}{rrrrrrrrr}
\hline$y$ & Re $\sigma_{11}$ & \multicolumn{1}{c}{ diff. } & Re $\sigma_{12}$ & diff. & Re $\sigma_{21}$ & diff. & Re $\sigma_{22}$ & diff. \\
\hline 0.50 & $2.48178 \mathrm{e}-6$ & $4 \mathrm{e}-6$ & 1.032461 & $-3 \mathrm{e}-6$ & $5.86125 \mathrm{e}-6$ & $2 \mathrm{e}-5$ & $-2.48067 \mathrm{e}-6$ & $-4 \mathrm{e}-6$ \\
0.45 & $-9.64956 \mathrm{e}-3$ & $1 \mathrm{e}-5$ & 0.491544 & $-3 \mathrm{e}-5$ & $2.78447 \mathrm{e}-5$ & $-3 \mathrm{e}-5$ & $9.64956 \mathrm{e}-3$ & $-1 \mathrm{e}-5$ \\
0.40 & $-1.28604 \mathrm{e}-2$ & $-1 \mathrm{e}-5$ & -0.063074 & $-1 \mathrm{e}-5$ & $5.03067 \mathrm{e}-4$ & $-3 \mathrm{e}-5$ & $1.28604 \mathrm{e}-2$ & $1 \mathrm{e}-5$ \\
0.35 & $-1.10649 \mathrm{e}-2$ & $-8 \mathrm{e}-7$ & -0.636047 & $3 \mathrm{e}-6$ & $2.00467 \mathrm{e}-3$ & $1 \mathrm{e}-6$ & $1.10649 \mathrm{e}-2$ & $8 \mathrm{e}-7$ \\
0.30 & $-7.45985 \mathrm{e}-3$ & $-7 \mathrm{e}-7$ & -1.236989 & $-2 \mathrm{e}-7$ & $5.07271 \mathrm{e}-3$ & $-5 \mathrm{e}-7$ & $7.45985 \mathrm{e}-3$ & $7 \mathrm{e}-7$ \\
0.25 & $-6.54480 \mathrm{e}-3$ & $-2 \mathrm{e}-6$ & -1.882453 & $1 \mathrm{e}-6$ & $1.02910 \mathrm{e}-2$ & $2 \mathrm{e}-6$ & $6.54480 \mathrm{e}-3$ & $2 \mathrm{e}-6$ \\
0.20 & $-1.25916 \mathrm{e}-2$ & $-3 \mathrm{e}-6$ & -2.568509 & $-5 \mathrm{e}-6$ & $1.82442 \mathrm{e}-2$ & $-5 \mathrm{e}-6$ & $1.25916 \mathrm{e}-2$ & $3 \mathrm{e}-6$ \\
0.15 & $-2.72072 \mathrm{e}-2$ & $-3 \mathrm{e}-5$ & -3.222155 & $-2 \mathrm{e}-5$ & $2.92067 \mathrm{e}-2$ & $-5 \mathrm{e}-5$ & $2.72072 \mathrm{e}-2$ & $3 \mathrm{e}-5$ \\
0.10 & $-4.69949 \mathrm{e}-2$ & $-6 \mathrm{e}-6$ & -3.674417 & $4 \mathrm{e}-7$ & $4.27968 \mathrm{e}-2$ & $3 \mathrm{e}-6$ & $4.69949 \mathrm{e}-2$ & $6 \mathrm{e}-6$ \\
0.05 & $-6.42886 \mathrm{e}-2$ & $2 \mathrm{e}-6$ & -3.718485 & $-8 \mathrm{e}-7$ & $5.76498 \mathrm{e}-2$ & $-1 \mathrm{e}-5$ & $6.42886 \mathrm{e}-2$ & $-2 \mathrm{e}-6$ \\
0.00 & $-7.10339 \mathrm{e}-2$ & $2 \mathrm{e}-5$ & -3.230427 & $-2 \mathrm{e}-5$ & $7.14417 \mathrm{e}-2$ & $1 \mathrm{e}-5$ & $7.10339 \mathrm{e}-2$ & $-2 \mathrm{e}-5$ \\
-0.05 & $-6.36424 \mathrm{e}-2$ & $-5 \mathrm{e}-6$ & -2.250092 & $8 \mathrm{e}-5$ & $8.18655 \mathrm{e}-2$ & $3 \mathrm{e}-4$ & $6.36424 \mathrm{e}-2$ & $5 \mathrm{e}-6$ \\
-0.10 & $-4.33228 \mathrm{e}-2$ & $3 \mathrm{e}-5$ & -0.947423 & $7 \mathrm{e}-5$ & $8.72315 \mathrm{e}-2$ & $1 \mathrm{e}-4$ & $4.33228 \mathrm{e}-2$ & $-3 \mathrm{e}-5$ \\
-0.15 & $-1.43936 \mathrm{e}-2$ & $1 \mathrm{e}-5$ & 0.454948 & $-1 \mathrm{e}-5$ & $8.69017 \mathrm{e}-2$ & $-7 \mathrm{e}-5$ & $1.43937 \mathrm{e}-2$ & $-1 \mathrm{e}-5$ \\
-0.20 & $1.81127 \mathrm{e}-2$ & $7 \mathrm{e}-7$ & 1.745284 & $1 \mathrm{e}-5$ & $8.10204 \mathrm{e}-2$ & $1 \mathrm{e}-5$ & $-1.81126 \mathrm{e}-2$ & $-7 \mathrm{e}-7$ \\
-0.25 & $4.94018 \mathrm{e}-2$ & $-9 \mathrm{e}-7$ & 2.762014 & $7 \mathrm{e}-6$ & $7.04199 \mathrm{e}-2$ & $5 \mathrm{e}-6$ & $-4.94019 \mathrm{e}-2$ & $9 \mathrm{e}-7$ \\
-0.30 & $7.62853 \mathrm{e}-2$ & $3 \mathrm{e}-6$ & 3.417222 & $2 \mathrm{e}-5$ & $5.67481 \mathrm{e}-2$ & $8 \mathrm{e}-5$ & $-7.62853 \mathrm{e}-2$ & $-3 \mathrm{e}-6$ \\
-0.35 & $9.68530 \mathrm{e}-2$ & $-2 \mathrm{e}-5$ & 3.682948 & $3 \mathrm{e}-4$ & $4.11869 \mathrm{e}-2$ & $2 \mathrm{e}-5$ & $-9.68530 \mathrm{e}-2$ & $2 \mathrm{e}-5$ \\
-0.40 & $9.63672 \mathrm{e}-2$ & $5 \mathrm{e}-6$ & 3.741658 & $-1 \mathrm{e}-5$ & $2.43069 \mathrm{e}-2$ & $-2 \mathrm{e}-6$ & $-9.63672 \mathrm{e}-2$ & $-5 \mathrm{e}-6$ \\
-0.45 & $5.23716 \mathrm{e}-2$ & $8 \mathrm{e}-7$ & 4.177614 & $-7 \mathrm{e}-6$ & $7.94868 \mathrm{e}-3$ & $7 \mathrm{e}-7$ & $-5.23716 \mathrm{e}-2$ & $-8 \mathrm{e}-7$ \\
-0.50 & $1.26826 \mathrm{e}-5$ & $9 \mathrm{e}-8$ & 5.152479 & $-1 \mathrm{e}-5$ & $1.05529 \mathrm{e}-5$ & $-8 \mathrm{e}-7$ & $-1.26824 \mathrm{e}-5$ & $-9 \mathrm{e}-8$ \\
\hline
\end{tabular}

Table A.8: Back-facing step after 13 refinements, derivative data for $\mathrm{x}=7$. The values in the "diff." columns are the differences between the value after 13 refinements and the value after 12 refinements. Recall that $\frac{\partial u_{i}}{\partial x_{j}}=\operatorname{Re} \sigma_{i j}$.

\begin{tabular}{rrrrrrrrr}
\hline$y$ & $u_{1}$ & diff. & $u_{2}$ & diff. & $p$ & diff. & $\omega$ & diff. \\
\hline 0.50 & $-8 \mathrm{e}-6$ & $-2 \mathrm{e}-9$ & $1.55645 \mathrm{e}-6$ & $4 \mathrm{e}-10$ & 0.207072 & $1 \mathrm{e}-6$ & 2.028947 & $4 \mathrm{e}-6$ \\
0.45 & 0.101024 & $-2 \mathrm{e}-8$ & $2.05568 \mathrm{e}-4$ & $3 \mathrm{e}-9$ & 0.207062 & $1 \mathrm{e}-6$ & 2.015290 & $2 \mathrm{e}-6$ \\
0.40 & 0.201929 & $-2 \mathrm{e}-8$ & $7.23070 \mathrm{e}-4$ & $-1 \mathrm{e}-8$ & 0.207056 & $1 \mathrm{e}-6$ & 2.024992 & $-5 \mathrm{e}-6$ \\
0.35 & 0.303943 & $-8 \mathrm{e}-8$ & $1.39643 \mathrm{e}-3$ & $-3 \mathrm{e}-8$ & 0.207051 & $1 \mathrm{e}-6$ & 2.057593 & $-3 \mathrm{e}-5$ \\
0.30 & 0.407653 & $1 \mathrm{e}-7$ & $2.07157 \mathrm{e}-3$ & $-4 \mathrm{e}-7$ & 0.207046 & $1 \mathrm{e}-6$ & 2.086530 & $-3 \mathrm{e}-5$ \\
0.25 & 0.511811 & $-5 \mathrm{e}-6$ & $2.60300 \mathrm{e}-3$ & $1 \mathrm{e}-6$ & 0.207041 & $2 \mathrm{e}-6$ & 2.066293 & $4 \mathrm{e}-4$ \\
0.20 & 0.612679 & $-9 \mathrm{e}-6$ & $2.87840 \mathrm{e}-3$ & $-5 \mathrm{e}-7$ & 0.207034 & $1 \mathrm{e}-6$ & 1.947035 & $-3 \mathrm{e}-4$ \\
0.15 & 0.704280 & $9 \mathrm{e}-6$ & $2.83166 \mathrm{e}-3$ & $2 \mathrm{e}-7$ & 0.207027 & $1 \mathrm{e}-6$ & 1.691098 & $2 \mathrm{e}-4$ \\
0.10 & 0.779349 & $-3 \mathrm{e}-8$ & $2.45438 \mathrm{e}-3$ & $1 \mathrm{e}-7$ & 0.207020 & $9 \mathrm{e}-7$ & 1.286676 & $4 \mathrm{e}-4$ \\
0.05 & 0.830852 & $1 \mathrm{e}-7$ & $1.79590 \mathrm{e}-3$ & $-3 \mathrm{e}-8$ & 0.207013 & $1 \mathrm{e}-6$ & 0.753618 & $1 \mathrm{e}-6$ \\
0.00 & 0.853412 & $2 \mathrm{e}-7$ & $9.51525 \mathrm{e}-4$ & $-1 \mathrm{e}-8$ & 0.207008 & $1 \mathrm{e}-6$ & 0.139054 & $-4 \mathrm{e}-5$ \\
-0.05 & 0.844427 & $1 \mathrm{e}-7$ & $4.12735 \mathrm{e}-5$ & $-1 \mathrm{e}-8$ & 0.207005 & $1 \mathrm{e}-6$ & -0.496125 & $-1 \mathrm{e}-6$ \\
-0.10 & 0.804490 & $9 \mathrm{e}-9$ & $-8.05745 \mathrm{e}-4$ & $-1 \mathrm{e}-8$ & 0.207005 & $1 \mathrm{e}-6$ & -1.087672 & $-1 \mathrm{e}-6$ \\
-0.15 & 0.737347 & $-6 \mathrm{e}-8$ & $-1.46760 \mathrm{e}-3$ & $-8 \mathrm{e}-9$ & 0.207006 & $1 \mathrm{e}-6$ & -1.577047 & $-7 \mathrm{e}-7$ \\
-0.20 & 0.649133 & $-9 \mathrm{e}-8$ & $-1.84905 \mathrm{e}-3$ & $-8 \mathrm{e}-9$ & 0.207009 & $1 \mathrm{e}-6$ & -1.926231 & $-8 \mathrm{e}-7$ \\
-0.25 & 0.547161 & $-1 \mathrm{e}-7$ & $-1.90766 \mathrm{e}-3$ & $-8 \mathrm{e}-9$ & 0.207012 & $1 \mathrm{e}-6$ & -2.127574 & $-1 \mathrm{e}-6$ \\
-0.30 & 0.438334 & $-2 \mathrm{e}-7$ & $-1.66320 \mathrm{e}-3$ & $-5 \mathrm{e}-9$ & 0.207016 & $1 \mathrm{e}-6$ & -2.206664 & $-8 \mathrm{e}-7$ \\
-0.35 & 0.327752 & $-2 \mathrm{e}-7$ & $-1.19613 \mathrm{e}-3$ & $-7 \mathrm{e}-9$ & 0.207019 & $1 \mathrm{e}-6$ & -2.207931 & $-4 \mathrm{e}-6$ \\
-0.40 & 0.217899 & $-1 \mathrm{e}-7$ & $-6.48459 \mathrm{e}-4$ & $6 \mathrm{e}-9$ & 0.207022 & $1 \mathrm{e}-6$ & -2.185100 & $3 \mathrm{e}-6$ \\
-0.45 & 0.108970 & $-8 \mathrm{e}-8$ & $-1.90603 \mathrm{e}-4$ & $8 \mathrm{e}-9$ & 0.207028 & $1 \mathrm{e}-6$ & -2.175421 & $2 \mathrm{e}-6$ \\
-0.50 & $-4 \mathrm{e}-6$ & $-2 \mathrm{e}-8$ & $-1.36906 \mathrm{e}-6$ & $-3 \mathrm{e}-10$ & 0.207037 & $1 \mathrm{e}-6$ & -2.186682 & $1 \mathrm{e}-5$ \\
\hline
\end{tabular}

Table A.9: Back-facing step after 13 refinements, data for $x=15$. The values in the "diff." columns are the differences between the value after 13 refinements and the value after 12 refinements. 


\begin{tabular}{rrrrrrrrr}
\hline$y$ & Re $\sigma_{11}$ & diff. & Re $\sigma_{12}$ & diff. & Re $\sigma_{21}$ & diff. & Re $\sigma_{22}$ & diff. \\
\hline 0.50 & $1.39416 \mathrm{e}-4$ & $-7 \mathrm{e}-7$ & -2.029033 & $2 \mathrm{e}-6$ & $-8.62423 \mathrm{e}-5$ & $5 \mathrm{e}-6$ & $-1.39394 \mathrm{e}-4$ & $7 \mathrm{e}-7$ \\
0.45 & $7.77970 \mathrm{e}-3$ & $-2 \mathrm{e}-7$ & -2.015385 & $5 \mathrm{e}-7$ & $-9.51861 \mathrm{e}-5$ & $3 \mathrm{e}-6$ & $-7.77970 \mathrm{e}-3$ & $2 \mathrm{e}-7$ \\
0.40 & $1.24104 \mathrm{e}-2$ & $-4 \mathrm{e}-7$ & -2.025009 & $-8 \mathrm{e}-7$ & $-1.73296 \mathrm{e}-5$ & $-6 \mathrm{e}-6$ & $-1.24104 \mathrm{e}-2$ & $4 \mathrm{e}-7$ \\
0.35 & $1.39684 \mathrm{e}-2$ & $5 \mathrm{e}-6$ & -2.057796 & $-2 \mathrm{e}-6$ & $-2.03503 \mathrm{e}-4$ & $-3 \mathrm{e}-5$ & $-1.39684 \mathrm{e}-2$ & $-5 \mathrm{e}-6$ \\
0.30 & $1.25228 \mathrm{e}-2$ & $1 \mathrm{e}-5$ & -2.086705 & $-6 \mathrm{e}-5$ & $-1.75105 \mathrm{e}-4$ & $-9 \mathrm{e}-5$ & $-1.25228 \mathrm{e}-2$ & $-1 \mathrm{e}-5$ \\
0.25 & $8.50853 \mathrm{e}-3$ & $3 \mathrm{e}-4$ & -2.066768 & $-1 \mathrm{e}-4$ & $-4.74536 \mathrm{e}-4$ & $2 \mathrm{e}-4$ & $-8.50851 \mathrm{e}-3$ & $-3 \mathrm{e}-4$ \\
0.20 & $2.41268 \mathrm{e}-3$ & $9 \mathrm{e}-5$ & -1.947766 & $-2 \mathrm{e}-4$ & $-7.30215 \mathrm{e}-4$ & $-5 \mathrm{e}-4$ & $-2.41266 \mathrm{e}-3$ & $-9 \mathrm{e}-5$ \\
0.15 & $-4.31467 \mathrm{e}-3$ & $-4 \mathrm{e}-5$ & -1.691568 & $3 \mathrm{e}-4$ & $-4.70322 \mathrm{e}-4$ & $5 \mathrm{e}-4$ & $4.31467 \mathrm{e}-3$ & $4 \mathrm{e}-5$ \\
0.10 & $-1.05554 \mathrm{e}-2$ & $-2 \mathrm{e}-5$ & -1.287243 & $-1 \mathrm{e}-4$ & $-5.67578 \mathrm{e}-4$ & $2 \mathrm{e}-4$ & $1.05554 \mathrm{e}-2$ & $2 \mathrm{e}-5$ \\
0.05 & $-1.54080 \mathrm{e}-2$ & $7 \mathrm{e}-7$ & -0.754515 & $1 \mathrm{e}-6$ & $-8.96537 \mathrm{e}-4$ & $3 \mathrm{e}-6$ & $1.54080 \mathrm{e}-2$ & $-7 \mathrm{e}-7$ \\
0.00 & $-1.81478 \mathrm{e}-2$ & $-7 \mathrm{e}-6$ & -0.139208 & $2 \mathrm{e}-5$ & $-1.53458 \mathrm{e}-4$ & $-2 \mathrm{e}-5$ & $1.81477 \mathrm{e}-2$ & $7 \mathrm{e}-6$ \\
-0.05 & $-1.80164 \mathrm{e}-2$ & $2 \mathrm{e}-7$ & 0.496776 & $1 \mathrm{e}-6$ & $6.51222 \mathrm{e}-4$ & $-1 \mathrm{e}-7$ & $1.80163 \mathrm{e}-2$ & $-2 \mathrm{e}-7$ \\
-0.10 & $-1.53944 \mathrm{e}-2$ & $-8 \mathrm{e}-10$ & 1.087924 & $2 \mathrm{e}-6$ & $2.52272 \mathrm{e}-4$ & $3 \mathrm{e}-7$ & $1.53944 \mathrm{e}-2$ & $1 \mathrm{e}-9$ \\
-0.15 & $-1.06589 \mathrm{e}-2$ & $3 \mathrm{e}-8$ & 1.577316 & $9 \mathrm{e}-7$ & $2.69271 \mathrm{e}-4$ & $2 \mathrm{e}-7$ & $1.06589 \mathrm{e}-2$ & $-3 \mathrm{e}-8$ \\
-0.20 & $-4.49000 \mathrm{e}-3$ & $-1 \mathrm{e}-8$ & 1.926515 & $7 \mathrm{e}-7$ & $2.84392 \mathrm{e}-4$ & $-4 \mathrm{e}-8$ & $4.48999 \mathrm{e}-3$ & $1 \mathrm{e}-8$ \\
-0.25 & $2.16201 \mathrm{e}-3$ & $-1 \mathrm{e}-8$ & 2.128026 & $1 \mathrm{e}-6$ & $4.51872 \mathrm{e}-4$ & $-2 \mathrm{e}-7$ & $-2.16199 \mathrm{e}-3$ & $7 \mathrm{e}-9$ \\
-0.30 & $7.50650 \mathrm{e}-3$ & $2 \mathrm{e}-7$ & 2.206833 & $5 \mathrm{e}-7$ & $1.69042 \mathrm{e}-4$ & $-3 \mathrm{e}-7$ & $-7.50650 \mathrm{e}-3$ & $-2 \mathrm{e}-7$ \\
-0.35 & $1.06464 \mathrm{e}-2$ & $-4 \mathrm{e}-7$ & 2.208253 & $-3 \mathrm{e}-7$ & $3.22138 \mathrm{e}-4$ & $-5 \mathrm{e}-6$ & $-1.06464 \mathrm{e}-2$ & $4 \mathrm{e}-7$ \\
-0.40 & $1.06249 \mathrm{e}-2$ & $3 \mathrm{e}-7$ & 2.185228 & $-1 \mathrm{e}-6$ & $1.27105 \mathrm{e}-4$ & $2 \mathrm{e}-6$ & $-1.06249 \mathrm{e}-2$ & $-3 \mathrm{e}-7$ \\
-0.45 & $7.10657 \mathrm{e}-3$ & $-1 \mathrm{e}-7$ & 2.175527 & $-2 \mathrm{e}-6$ & $1.05887 \mathrm{e}-4$ & $4 \mathrm{e}-7$ & $-7.10657 \mathrm{e}-3$ & $1 \mathrm{e}-7$ \\
-0.50 & $1.70887 \mathrm{e}-4$ & $1 \mathrm{e}-6$ & 2.186746 & $-3 \mathrm{e}-6$ & $6.33213 \mathrm{e}-5$ & $7 \mathrm{e}-6$ & $-1.70857 \mathrm{e}-4$ & $-1 \mathrm{e}-6$ \\
\hline
\end{tabular}

Table A.10: Back-facing step after 13 refinements, derivative data for $\mathrm{x}=15$. The values in the "diff." columns are the differences between the value after 13 refinements and the value after 12 refinements. Recall that $\frac{\partial u_{i}}{\partial x_{j}}=\operatorname{Re} \sigma_{i j}$. 


\section{References}

[1] Andrew T. Barker, Susanne C. Brenner, Eun-Hee Park, and Li-Yeng Sung. A one-level additive Schwarz preconditioner for a discontinuous Petrov-Galerkin method. http: / / arxiv. org/abs/1212.2645, December 2012.

[2] Pavel Bochev and R. B. Lehoucq. On the finite element solution of the pure Neumann problem. SIAM Review, 47(1):55-66, March 2005.

[3] O. Botella and R. Peyret. Benchmark spectral results on the lid-driven cavity flow. Computers \& Fluids, 27(4):421-433, 1998.

[4] C.L. Bottasso, S. Micheletti, and R. Sacco. The discontinuous Petrov-Galerkin method for elliptic problems. Comput. Methods Appl. Mech. Engrg., 191:3391-3409, 2002.

[5] C.L. Bottasso, S. Micheletti, and R. Sacco. A multiscale formulation of the discontinuous Petrov-Galerkin method for advective-diffusive problems. Comput. Methods Appl. Mech. Engrg., 194:2819-2838, 2005.

[6] J. Bramwell, L. Demkowicz, and W. Qiu. Solution of dual-mixed elasticity equations using Arnold-Falk-Winther element and discontinuous Petrov-Galerkin method, a comparison. Technical Report 2010-23, ICES, 2010.

[7] Dirk Broersen and Rob P. Stevenson. A petrov-galerkin discretization with optimal test space of a mild-weak formulation of convectiondiffusion equations in mixed form. IMA Journal of Numerical Analysis, 2014.

[8] Charles-Henri Bruneau and Mazen Saad. The 2D lid-driven cavity problem revisited. Computers \& Fluids, 35(3):326 - 348, 2006.

[9] Tan Bui-Thanh, Leszek Demkowicz, and Omar Ghattas. A unified discontinuous Petrov-Galerkin method and its analysis for Friedrichs' systems. Submitted to SIAM J. Numer. Anal., 2011. Also ICES report ICES-11-34, November 2011.

[10] Tan Bui-Thanh and Omar Ghattas. A PDE-constrained optimization approach to the discontinuous Petrov-Galerkin method with a trust region inexact Newton-CG solver. Technical Report 13-16, ICES, 2013.

[11] Donna Calhoun. A Cartesian grid method for solving the two-dimensional streamfunction-vorticity equations in irregular regions. Journal of Computational Physics, 176(2):231-275, 2002.

[12] J. Chan, L. Demkowicz, and R. Moser. A DPG method for steady viscous compressible flow. 13-23, ICES, https://www.ices.utexas.edu/media/reports/2013/1323.pdf, 2013.

[13] J. Chan, L. Demkowicz, R. Moser, and N. Roberts. A class of Discontinuous Petrov-Galerkin methods. Part V: Solution of 1D Burgers and Navier-Stokes equations. Technical Report 25, ICES, 2010.

[14] J. Chan, L. Demkowicz, and M. Shashkov. Space-time DPG for shock problems. Technical Report Technical Report LA-UR 11-05511, LANL, September 2011

[15] Madeleine Coutanceau and Roger Bouard. Experimental determination of the main features of the viscous flow in the wake of a circular cylinder in uniform translation. part 1. steady flow. Journal of Fluid Mechanics, 79(02):231-256, 1977.

[16] W. Dahmen, A. Cohen, and G. Welper. Adaptivity and variational stabilization for convection-diffusion equations. ESAIM: Mathematical Modelling and Numerical Analysis, 46(5):1247-1273, 2012.

[17] L. Demkowicz. Computing with hp Finite Elements. I. One- and Two-Dimensional Elliptic and Maxwell Problems. Chapman \& Hall/CRC Press, Taylor and Francis, October 2006.

[18] L. Demkowicz and J. Gopalakrishnan. A class of discontinuous Petrov-Galerkin methods. Part I: The transport equation. Comput. Methods Appl. Mech. Engrg., 199:1558-1572, 2010. See also ICES Report 2009-12.

[19] L. Demkowicz and J. Gopalakrishnan. Analysis of the DPG method for the Poisson problem. SIAM J. Num. Anal., 49(5):1788-1809, 2011.

[20] L. Demkowicz and J. Gopalakrishnan. A class of discontinuous Petrov-Galerkin methods. Part II: Optimal test functions. Numer. Meth. Part. D. E., 27(1):70-105, January 2011.

[21] L. Demkowicz and N. Heuer. Robust DPG method for convection-dominated diffusion problems. Technical Report 33 , ICES, 2011.

[22] L. Demkowicz, J. Kurtz, D. Pardo, M. Paszyński, W. Rachowicz, and A. Zdunek. Computing with hp Finite Elements. II. Frontiers: ThreeDimensional Elliptic and Maxwell Problems with Applications. Chapman \& Hall/CRC, October 2007.

[23] SCR Dennis and Gau-Zu Chang. Numerical solutions for steady flow past a circular cylinder at reynolds numbers up to 100. J. Fluid Mech, 42(3):471-489, 1970

[24] Bengt Fornberg. A numerical study of steady viscous flow past a circular cylinder. Journal of Fluid Mechanics, 98(4):819-855, 1980.

[25] David K. Gartling. A test problem for outflow boundary conditions-flow over a backward-facing step. International Journal for Numerical Methods in Fluids, 11(7):953-967, 1990.

[26] Jay Gopalakrishnan and Weifeng Qiu. An analysis of the practical DPG method. Mathematics of Computation, 2012.

[27] W.J. Gordon and C.A. Hall. Transfinite element methods: blending function interpolation over arbitrary curved element domain. Numer. Math., 21:109-129, 1973.

[28] Xiaoyi He and Gary Doolen. Lattice Boltzmann method on curvilinear coordinates system: flow around a circular cylinder. Journal of Computational Physics, 134(2):306-315, 1997.

[29] Michael A. Heroux, Roscoe A. Bartlett, Vicki E. Howle, Robert J. Hoekstra, Jonathan J. Hu, Tamara G. Kolda, Richard B. Lehoucq, Kevin R. Long, Roger P. Pawlowski, Eric T. Phipps, Andrew G. Salinger, Heidi K. Thornquist, Ray S. Tuminaro, James M. Willenbring, Alan Williams, and Kendall S. Stanley. An overview of the Trilinos project. ACM Trans. Math. Softw., 31(3):397-423, 2005.

[30] Jiten C. Kalita and Shuvam Sen. Triggering asymmetry for flow past circular cylinder at low Reynolds numbers. Computers \& Fluids, 59(0):44 - 60, 2012

[31] G. Karniadakis and S. Sherwin. Spectral/hp Element Methods for Computational Fluid Dynamics. Oxford Science Publications, 2nd edition, 2005 .

[32] L. I. G. Kovasznay. Laminar flow behind a two-dimensional grid. Mathematical Proceedings of the Cambridge Philosophical Society, 44(01):58-62, 1948.

[33] L.S.G. Kovasznay. Hot-wire investigation of the wake behind cylinders at low Reynolds numbers. Proceedings of the Royal Society of London. Series A, Mathematical and Physical Sciences, 198(1053):174-190, 1949.

[34] DV Le, BC Khoo, and J Peraire. An immersed interface method for viscous incompressible flows involving rigid and flexible boundaries. Journal of Computational Physics, 220(1):109-138, 2006. 
[35] Ta Phuoc Loc. Etude numerique de l'ecoulement d'un fluide visqueux incompressible autour d'un cylindre fixe ou en rotation, effet magnus. J. Mec, 14(1):109-134, 1975.

[36] Ta Phuoc Loc. Numerical analysis of unsteady secondary vortices generated by an impulsively started circular cylinder. Journal of Fluid Mechanics, 100(1):111-128, 1980.

[37] H.K. Moffatt. Viscous and resistive eddies near a sharp corner. Journal of Fluid Mechanics, 18(1):1-18, 1964.

[38] D. Moro, N.C. Nguyen, and J. Peraire. A hybridized discontinuous Petrov-Galerkin scheme for scalar conservation laws. Int.J. Num. Meth. Eng., 2011. in print.

[39] A.H. Niemi, J.A. Bramwell, and L.F. Demkowicz. Discontinuous Petrov-Galerkin method with optimal test functions for thin-body problems in solid mechanics. Computer Methods in Applied Mechanics and Engineering, 200(9-12):1291-1300, Feb 2011.

[40] F Nieuwstadt and HB Keller. Viscous flow past circular cylinders. Computers \& Fluids, 1(1):59-71, 1973.

[41] XD Niu, YT Chew, and C Shu. Simulation of flows around an impulsively started circular cylinder by taylor series expansion-and least squares-based lattice Boltzmann method. Journal of Computational Physics, 188(1):176-193, 2003.

[42] J.T. Oden. Error estimation and control in computational fluid dynamics. In J. Whiteman, editor, The Mathematics of Finite Elements and Applications: Highlights 1993, volume 8, pages 1-23. John Wiley \& Sons, 1993.

[43] A. Ozcelikkale and C. Sert. Least-squares spectral element solution of incompressible navier-stokes equations with adaptive refinement. $J$. Comput. Physics, 231(9):3755-3769, 2012.

[44] JP Pontaza and JN Reddy. Spectral/hp least-squares finite element formulation for the Navier-Stokes equations. Journal of Computational Physics, 190(2):523-549, 2003.

[45] V Prabhakar and JN Reddy. Spectral/hp penalty least-squares finite element formulation for the steady incompressible Navier-Stokes equations. Journal of Computational Physics, 215(1):274-297, 2006.

[46] Nathan V. Roberts. A Discontinuous Petrov-Galerkin Methodology for Incompressible Flow Problems. PhD thesis, University of Texas at Austin, 2013.

[47] Nathan V. Roberts. Camellia: A software framework for discontinuous Petrov-Galerkin methods. Computers \& Mathematics with Applications, 2014.

[48] Nathan V. Roberts, Tan Bui-Thanh, and Leszek F. Demkowicz. The DPG method for the Stokes problem. Computers and Mathematics with Applications, 2014.

[49] Nathan V. Roberts, Denis Ridzal, Pavel B. Bochev, Leszek Demkowicz, Kara J. Peterson, and Christopher M. Siefert. Application of a discontinuous Petrov-Galerkin method to the Stokes equations. In CSRI Summer Proceedings 2010. Sandia National Laboratories, 2010.

[50] David Russell and Z Jane Wang. A Cartesian grid method for modeling multiple moving objects in 2D incompressible viscous flow. Journal of Computational Physics, 191(1):177-205, 2003.

[51] YVSS Sanyasiraju and V Manjula. Flow past an impulsively started circular cylinder using a higher-order semicompact scheme. Physical Review E, 72(1):016709, 2005.

[52] Hideo Takami and Herbert B. Keller. Steady two-dimensional viscous flow of an incompressible fluid past a circular cylinder. Physics of Fluids, 12(12):II-51-II-56, 1969.

[53] DJ Tritton. Experiments on the flow past a circular cylinder at low Reynolds numbers. J. Fluid Mech, 6(4):547-567, 1959.

[54] C. Wieners and B. Wohlmuth. Robust operator estimates and the application to sub structuring methods for first-order systems. ESAIM: Mathematical Modelling and Numerical Analysis, 48(5):1473-1494, 2014.

[55] Tao Ye, Rajat Mittal, HS Udaykumar, and Wei Shyy. An accurate Cartesian grid method for viscous incompressible flows with complex immersed boundaries. Journal of Computational Physics, 156(2):209-240, 1999.

[56] J. Zitelli, I. Muga, L. Demkowicz, J. Gopalakrishnan, D. Pardo, and V. Calo. A class of discontinuous Petrov-Galerkin methods. Part IV: Wave propagation problems. J. Comp. Phys., 230:2406-2432, 2011. 